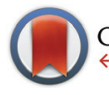

CrossMark click for updates

Cite this: Org. Biomol. Chem., 2014,

Received 4th June 2014, Accepted 7th July 2014

DOI: 10.1039/c4ob01151a

www.rsc.org/obc 12,6490

\section{Total synthesis of the cyclic monoterpenoid pyrano[3,2-a]carbazole alkaloids derived from 2-hydroxy-6-methylcarbazole $\uparrow+$}

\author{
Cemena Gassner, Ronny Hesse, Arndt W. Schmidt and Hans-Joachim Knölker*
}

The synthesis of seven pyrano[3,2-a]carbazole alkaloids has been achieved using their putative biogenetic precursor 2-hydroxy-6-methylcarbazole as key intermediate.

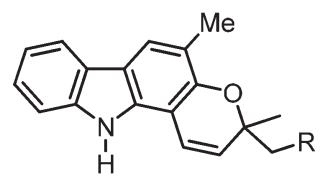

1 Girinimbine $\mathrm{R}=\mathrm{H}$

2 Mahanimbine $\mathrm{R}=$ prenyl

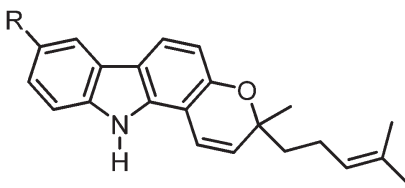

(+)-4 Mahanimbicine $\mathrm{R}=\mathrm{Me}$

$(-)-4$ Isomahanimbine $\mathrm{R}=\mathrm{Me}$ 5 Murrayamine-J $\mathrm{R}=\mathrm{CHO}$

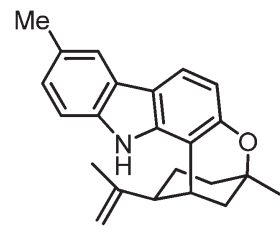

8 Murrayamine-G

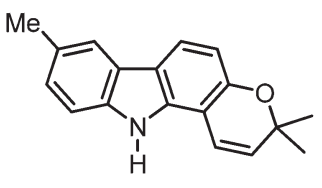

3 Isogirinimbine

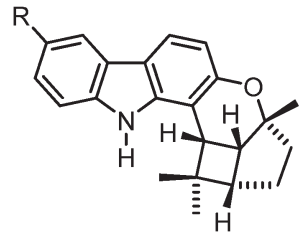

6 Bicyclomahanimbicine $\mathrm{R}=\mathrm{Me}$ 7 Murrayamine-M $\quad \mathrm{R}=\mathrm{CHO}$

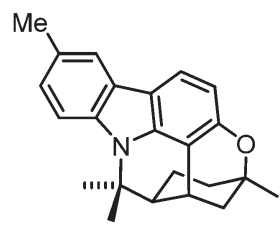

9 Isomurrayazoline fusion of 2-hydroxy-6-methylcarbazole (10) and geranyl diphosphate were isolated from natural sources. ${ }^{2}$ In 1970, Kapil et al. isolated mahanimbicine $[(+)-4]$ and bicyclomahanimbicine (6) from Murraya koenigii. ${ }^{5}$ Subsequently, Crombie and Whiting et al. proposed the correct structure for bicyclomahanimbicine (6). ${ }^{6}$ It is interesting to note that also in 1970 , Joshi et al. obtained (-)-4 from the leaves of the same plant and named it isomahanimbine, ${ }^{7}$ however, the absolute configuration was not determined. The structures of $\mathbf{4}$ and $\mathbf{6}$ were supported by synthesis. $^{4 c, d, 5}$

Department Chemie, Technische Universität Dresden, Bergstrasse 66, 01069 Dresden, Germany. E-mail: hans-joachim. knoelker@tu-dresden.de; Fax: +49 351 463-37030 $\dagger$ Part 121 of Transition Metals in Organic Synthesis; for Part 120, see ref. 14c. $\$$ Electronic supplementary information (ESI) available: ${ }^{1} \mathrm{H}$ and ${ }^{13} \mathrm{C}$ NMR spectra for all compounds. CCDC 1000251. For ESI and crystallographic data in CIF or other electronic format see DOI: 10.1039/c4ob01151a
Fig. 1 Naturally occurring pyrano[3,2-a]carbazole alkaloids 1-9.

Wu et al. isolated murrayamine-J $(5),{ }^{8}$ murrayamine-M $(7)^{8}$ and murrayamine-G (8), ${ }^{9}$ from the leaves of Murraya euchrestifolia. The hexacyclic pyrano[3,2-a]carbazole alkaloid isomurrayazoline (9) was obtained in 1982 by Chakraborty et al. from the stem bark of Murraya koenigii. ${ }^{10}$

We have developed diverse synthetic approaches to pyrano[3,2-a]carbazoles including girinimbine (1), mahanimbine (2), pyrayafoline $\mathrm{A}-\mathrm{E}$ and monoterpenoid pyrano[3,2-a]carbazole alkaloids. ${ }^{11-14}$ Herein, we describe the synthesis of isogirinimbine (3), ( \pm )-mahanimbicine [( \pm -)-isomahanimbine] [( \pm$)-4]$, murrayamine-J (5) and the cyclic monoterpenoid pyrano[3,2-a]carbazole alkaloids $\mathbf{6 - 9}$, which biogenetically derive from 
<smiles>CC=CC</smiles><smiles>COc1cccc(Br)c1</smiles>
11

12

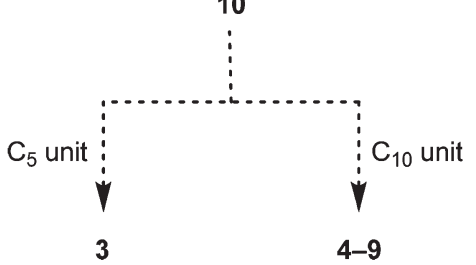

Scheme 1 Synthetic route to the pyrano[3,2-a]carbazoles 3-9.

2-hydroxy-6-methylcarbazole (10). Key steps of our approach are an efficient construction of the carbazole $\mathbf{1 0}$ based on our palladium-catalyzed route ${ }^{15}$ and a subsequent annulation of either a $\mathrm{C}_{5}$ or a $\mathrm{C}_{10}$ building block (Scheme 1). The substitution pattern present in compound $\mathbf{1 0}$ has been generated previously in our synthesis of 7-oxygenated carbazole alkaloids. $^{16}$

\section{Results and discussion}

Buchwald-Hartwig coupling of $p$-toluidine (11) and $m$-bromoanisole (12) in the presence of SPhos (2-dicyclohexylphosphino-2',6'-dimethoxybiphenyl) ${ }^{17}$ afforded the diarylamine 13 (Scheme 2). Alternatively, compound $\mathbf{1 3}$ has been prepared quantitatively on a $25 \mathrm{~g}$ scale by Buchwald-Hartwig coupling of $m$-anisidine and $p$-bromotoluene (see Experimental section). ${ }^{16}$ Palladium(II)-catalyzed oxidative cyclization of 13 provided the desired 2-methoxy-6-methylcarbazole $(\mathbf{1 4})^{16}$ as major product ( $89 \%$ yield) and up to $5 \%$ of glycoborine $(\mathbf{1 5})^{18}$ as by-product. Cleavage of the methyl ether led to 2-hydroxy-6methylcarbazole (10). Formation of the dimethylpropargyl ether via Godfrey's method, ${ }^{19}$ followed by a thermally induced sequence of Claisen rearrangement, 1,5-hydrogen shift and electrocyclic ring closure ${ }^{20}$ provided isogirinimbine (3) in $63 \%$ yield and as a by-product the furo[3,2-a]carbazole 17 in up to $3 \%$ yield. The structure of isogirinimbine (3) has been fully supported by its spectroscopic data which confirm it as an isomer of the natural product girinimbine (1). The 3-methylregioisomer of $\mathbf{1 7}$ was obtained previously as by-product in our synthesis of girinimbine (1). ${ }^{12}$

We envisaged ( \pm -mahanimbicine $[( \pm)$-isomahanimbine $]$ $[( \pm)-4]$ as crucial intermediate for the synthesis of the formyl derivative murrayamine-J (5) and the cyclic monoterpenoid pyrano[3,2-a]carbazole alkaloids 6-9. Thus, we have developed two alternative synthetic routes for the synthesis of $( \pm)-4$. The first approach requires no protecting group (Scheme 3). Reaction of 2-hydroxy-6-methylcarbazole (10) with the carbonate $18^{21}$ in the presence of catalytic amounts of copper(I) iodide and subsequent thermally induced rearrangement provided<smiles>COc1cccc(Nc2ccc(C)cc2)c1</smiles><smiles>COc1ccc2c(c1)[nH]c1ccc(C)cc12</smiles><smiles>[R]=CCCC</smiles>

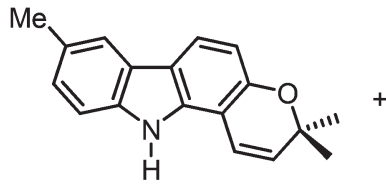

3 Isogirinimbine

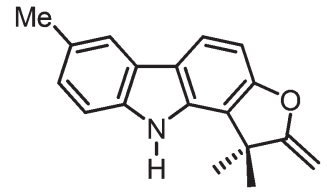

17
Scheme 2 Synthesis of isogirinimbine (3). Reagents and conditions: (a) 1.3 equiv. 11, 5 mol\% $\mathrm{Pd}(\mathrm{OAc})_{2}, 10 \mathrm{~mol} \%$ SPhos, 1.4 equiv. $\mathrm{Cs}_{2} \mathrm{CO}_{3}$, toluene, reflux, $17.5 \mathrm{~h}, 98 \%$; (b) $3 \mathrm{~mol} \% \mathrm{Pd}(\mathrm{OAc})_{2}, 10 \mathrm{~mol} \% \mathrm{~K}_{2} \mathrm{CO}_{3}$, PivOH, $100{ }^{\circ} \mathrm{C}, 20.5 \mathrm{~h}, 89 \% 14$ and $\leq 5 \% 15$; (c) 1.9 equiv. $\mathrm{BBr}_{3},-78{ }^{\circ} \mathrm{C}$ to rt, $2 \mathrm{~h}, 91 \%$; (d) 1.1 .1 equiv. 16, 1.1 equiv. TFAA, 2.8 equiv. DBU, $0.2 \mathrm{~mol} \%$ Cul, $\mathrm{MeCN},-15{ }^{\circ} \mathrm{C}$ to $\mathrm{rt}, 6.5 \mathrm{~h}$; 2 . toluene, reflux, $23 \mathrm{~h}, 63 \% 3$ and $\leq 3 \% 17$.<smiles>Cc1ccc2c(c1)c1ccc(O)cc1n2[18OH]</smiles>

$\mathrm{Me}$<smiles>CC(C)=CCCC1(C)C=Cc2c(ccc3c2[nH]c2ccc(F)cc23)O1</smiles>

$( \pm)-4$ ( \pm )-Mahanimbicine
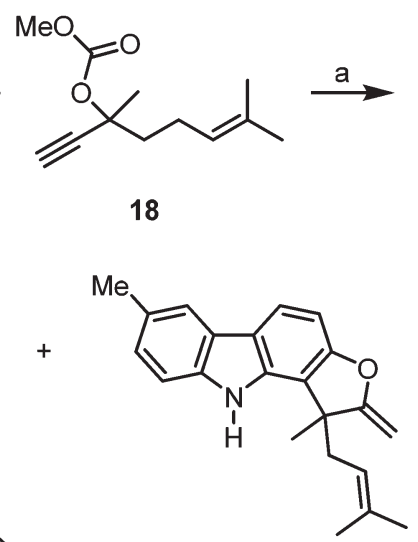

19
Scheme 3 Synthesis of $( \pm)$-mahanimbicine [( \pm )-4]. Reagents and conditions: (a) 1.1 .5 equiv. 18, 2.0 equiv. DBU, 0.5 mol\% Cul, MeCN, rt, $22 \mathrm{~h}$; 2. toluene, reflux, $22.5 \mathrm{~h}, 49 \%( \pm)-4$ and $\leq 5 \% 19$.

$( \pm)$-mahanimbicine $[( \pm)-4]$ in $49 \%$ yield along with the furo$[3,2-a]$ carbazole 19 in up to $5 \%$ yield as by-product.

Alternatively, 2-methoxy-6-methylcarbazole (14) was initially protected by transformation to the $N$-tosylcarbazole 20 


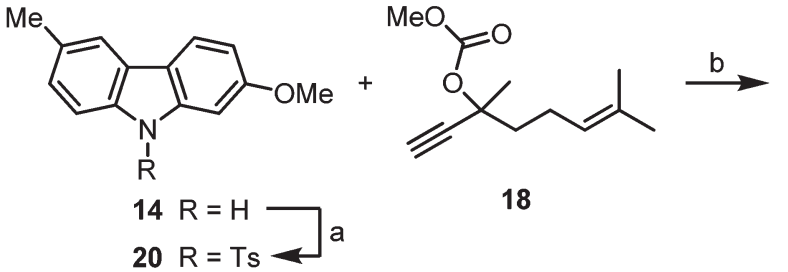<smiles>CC(C)=CCCC1(C)C=Cc2c(ccc3c4cc(C)ccc4n([Tl])c23)O1</smiles><smiles>COc1ccc2c(c1)c1cc3c(cc1n2C(F)(F)F)OC(C)(CCC=C(C)C)C=C3</smiles>

22<smiles>CC=C(C)CCC1(C)C=Cc2c(ccc3c2[nH]c2ccc(C)cc23)O1</smiles>

( \pm )-4 ( \pm )-Mahanimbicine

Scheme 4 Alternative route to $( \pm)$-mahanimbicine [( \pm )-4]. Reagents and conditions: (a) 4.1 equiv. $\mathrm{NaH}, 1.5$ equiv. $\mathrm{TsCl}, \mathrm{THF}, 0{ }^{\circ} \mathrm{C}$ to rt, $16.25 \mathrm{~h}, 80 \%$; (b) 1.3 .0 equiv. $\mathrm{BBr}_{3}, \mathrm{CH}_{2} \mathrm{Cl}_{2},-78{ }^{\circ} \mathrm{C}$ to rt, $2.5 \mathrm{~h}$; 2.2 .0 equiv. 18, 3.0 equiv. DBU, 0.5 mol\% Cul, $\mathrm{MeCN}, \mathrm{rt}, 22 \mathrm{~h} ; 3$. xylene, reflux, $27.5 \mathrm{~h}, 82 \%$ ( 3 steps, ratio $21 / 22=7.7: 1$ ); (c) 4.0 equiv. TBAF, THF, $75{ }^{\circ} \mathrm{C}$, $6 \mathrm{~h}, 79 \%( \pm)-4$ and $9 \% 23$.

(Scheme 4). Cleavage of the methyl ether, copper-catalyzed reaction with the carbonate $\mathbf{1 8}$ and thermal rearrangement led in $82 \%$ yield to a mixture of the pyrano[3,2-a]carbazole 21 and the pyrano[2,3-b]carbazole 22 in a ratio of $7.7: 1$. Finally, removal of the tosyl group by treatment with tetrabutylammonium fluoride ${ }^{22}$ at elevated temperature provided ( \pm -mahanimbicine $[( \pm)-4]$.

Using the first approach (Scheme 3), ( \pm )-mahanimbicine $[( \pm)-4]$ is available in 5 steps and $40 \%$ overall yield based on $p$-bromotoluene. Our second route (Scheme 4) leads to $( \pm)-\mathbf{4}$ in 7 steps and $46 \%$ overall yield based on the same starting material. It is interesting to note, that annulation of the pyran ring with the carbonate $\mathbf{1 8}$ at 2-hydroxy-6-methylcarbazole (10) provides the furo[3,2- $a$ carbazole 19 as by-product, whereas annulation at the corresponding $N$-tosylcarbazole gives the pyrano[2,3-b]carbazole 22 as by-product. This outcome is explained by the steric demand of the tosyl group which suppresses the formation of $N$-tosyl-19 with the quaternary carbon center in close proximity to the protecting group; instead linear pyran annulation and thus formation of compound 22 is observed.

Using $( \pm)$-mahanimbicine $[( \pm)-4]$ as relay compound the carbazole alkaloids 5-9 are accessible, following putative bio-

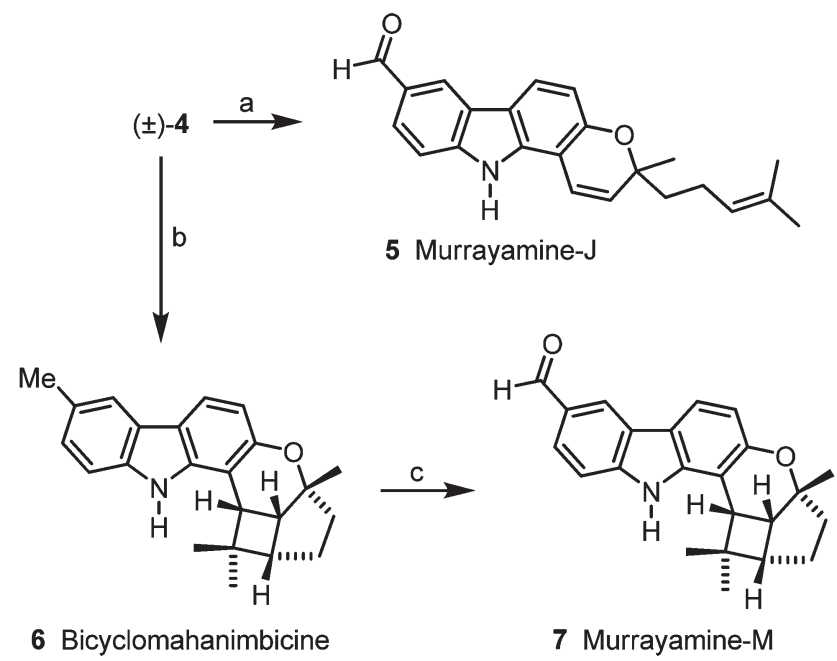

Scheme 5 Synthesis of murrayamine-J (5), bicyclomahanimbicine (6) and murrayamine-M (7). Reagents and conditions: (a) 8.0 equiv. DDQ, $\mathrm{MeOH}-\mathrm{THF}$-water $(10: 1: 1), \mathrm{rt}, 6.25 \mathrm{~h}, 67 \%$; (b) $h \nu$, toluene, rt, $14 \mathrm{~d}$, $39 \%$; (c) 6.6 equiv. DDQ, MeOH-THF-water (10:1:2), rt, 5 h, $51 \%$.

genetic routes. Oxidation of $( \pm)$-mahanimbicine $[( \pm)-4]$ with 2,3-dichloro-5,6-dicyano-1,4-benzoquinone (DDQ) afforded murrayamine-J (5) (Scheme 5). Intramolecular $[2+2]$ cycloaddition of $[( \pm)-4]$ led to bicyclomahanimbicine (6). Oxidation of 6 with DDQ gave murrayamine-M (7).

For the Brønsted acid promoted cycloisomerization of $( \pm)$-mahanimbicine $[( \pm)-4]$, we took advantage of our previous study on the conversion of mahanimbine (2) into cyclomahanimbine and mahanimbidine. ${ }^{12}$ On treatment of $( \pm)-4$ with one equivalent camphor-10-sulfonic acid (CSA) at room temperature to $70{ }^{\circ} \mathrm{C}$ for $16 \mathrm{~d},( \pm)-\mathbf{4}$ and rapidly formed 9 were both completely converted into 8. Thus, murrayamine-G (8) was obtained in $65 \%$ yield (Scheme 6 , Table 1 ). Cycloisomerization of ( \pm -mahanimbicine $[( \pm)-4]$ in the presence of catalytic amounts of CSA in hexane at room temperature afforded in $70 \%$ yield a $1: 1$ mixture of $\mathbf{8}$ and $\mathbf{9}$ which after separation by preparative HPLC led to pure isomurrayazoline (9). Our syn-

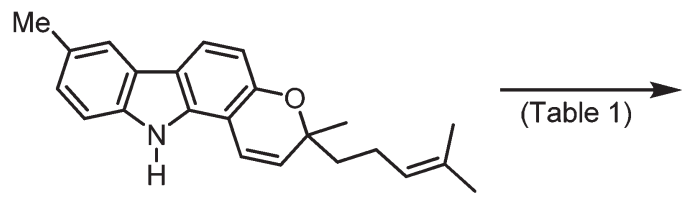

$( \pm)-4$ ( \pm )-Mahanimbicine

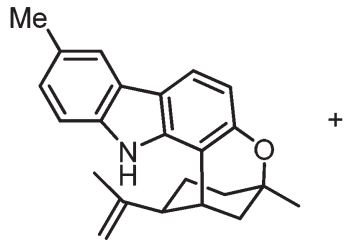

8 Murrayamine-G

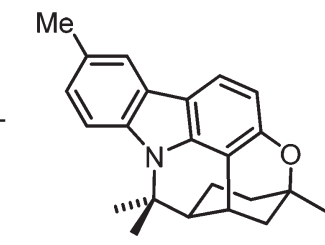

9 Isomurrayazoline
Scheme 6 Synthesis of murrayamine-G (8) and isomurrayazoline (9). Reagents and conditions: see Table 1. 
Table 1 Cycloisomerization of $( \pm)$-mahanimbicine $[( \pm)-4]$

\begin{tabular}{lll}
\hline Reaction conditions & Yield & Ratio, 8:9 \\
\hline 1.0 equiv. CSA, PhMe, rt to $70^{\circ} \mathrm{C}, 16 \mathrm{~d}$ & $65 \% \mathbf{8}$ & - \\
8 mol\% CSA, hexane, rt, $11.5 \mathrm{~d}$ & $70 \% \mathbf{8}, \mathbf{9}$ & $1: 1$
\end{tabular}

thetic route leads to isomurrayazoline (9) in 8 steps and $16 \%$ overall yield based on $p$-bromotoluene.

\section{Conclusions}

We have developed a highly efficient palladium-catalyzed route to 2-hydroxy-6-methylcarbazole (10) (3 steps, 81\% overall yield). Using appropriate $\mathrm{C}_{5}$ or $\mathrm{C}_{10}$ building blocks, compound $\mathbf{1 0}$ has been transformed into isogirinimbine (3) and ( \pm )-mahanimbicine $[( \pm)-\mathbf{4}]$, respectively. ( \pm )-Mahanimbicine $[( \pm)-\mathbf{4}]$ served as central intermediate for the synthesis of cyclic monoterpenoid pyrano[3,2-a]carbazole alkaloids and could be converted into murrayamine-J (5), bicyclomahanimbicine (6), murrayamine-M (7), murrayamine-G (8) and isomurrayazoline (9). The spectroscopic data of the alkaloids were in full agreement with those reported for the corresponding natural products. For the compounds 3, 5 and 7-9 we have achieved the first total synthesis. The present results emphasize the utility of our synthetic methodology and demonstrate that for the first time also the whole series of carbazole alkaloids which is isomeric to girinimbine (1) and mahanimbine (2) is available by synthesis on large scale. The biological properties of 3-9, e.g. their potential anti-TB activity, ${ }^{23}$ are under further investigation.

\section{Experimental}

\section{General methods}

All reactions were carried out in oven-dried glassware using dry solvents under an argon atmosphere unless stated otherwise. Acetonitrile, dichloromethane, tetrahydrofuran and toluene were dried using a solvent purification system (MBraun-SPS). Palladium(II) acetate was recrystallized from glacial acetic acid. All other chemicals were used as received from commercial sources. Flash chromatography was performed on a Büchi Sepacore system equipped with an UV monitor using silica gel from Acros Organics (0.035-0.070 $\mathrm{mm})$. Thin layer chromatography was performed with TLC plates from Merck $\left(60 \mathrm{~F}_{254}\right)$ using UV-light for visualization. Melting points were measured on a Gallenkamp MPD 350 melting point apparatus. Ultraviolet spectra were recorded on a Perkin Elmer 25 UV/VIS spectrometer. Infrared spectra were recorded on a Thermo Nicolet Avatar 360 FT-IR spectrometer using the ATR method (Attenuated Total Reflectance). NMR spectra were recorded on Bruker Avance II 300, DRX 500 and Avance III 600 spectrometers. Chemical shifts $\delta$ are reported in parts per million with the non-deuterated solvent as internal standard. ${ }^{24}$ The following abbreviations have been used: s: singlet, d: doublet, t: triplet, m: multiplet and br: broad. Mass spectra were recorded on a Finnigan MAT-95 spectrometer (electron impact, $70 \mathrm{eV}$ ) or by GC/MS-coupling using an Agilent Technologies 6890 N GC System equipped with a 5973 Mass Selective Detector (electron impact, $70 \mathrm{eV}$ ). ESI-MS spectra were recorded on an Esquire LC with an ion trap detector from Bruker. Positive and negative ions were detected. Elemental analyses were measured on an EuroVector EuroEA3000 elemental analyzer.

3-Methoxy- $\mathrm{N}$-(4-methylphenyl)aniline (13). Method A: A solution of $m$-bromoanisole (12) $(2.00 \mathrm{~g}, 10.7 \mathrm{mmol})$ in toluene $(5 \mathrm{~mL})$ was added dropwise over a period of $5 \mathrm{~h}$ to a suspension of $p$-toluidine (11) (1.51 g, $14.1 \mathrm{mmol})$, palladium(II) acetate (123 mg, $548 \mu \mathrm{mol}$ ), SPhos (440 mg, $1.07 \mathrm{mmol}$ ) and caesium carbonate $(4.90 \mathrm{~g}, 15.0 \mathrm{mmol})$ in toluene $(18 \mathrm{~mL})$ at reflux and the mixture was heated at reflux for $12.5 \mathrm{~h}$ (total reaction time: $17.5 \mathrm{~h}$ ). After cooling to room temperature, the mixture was filtered through a short pad of Celite (diethyl ether) and the solvent was evaporated. Purification of the residue by column chromatography on silica gel (pentanedichloromethane-ethyl acetate, $12: 5: 1$ ) provided 3-methoxy$N$-(4-methylphenyl)aniline (13) as colourless solid, yield: $2.24 \mathrm{~g}$ (98\%), m.p. $68-70{ }^{\circ} \mathrm{C}$. UV (MeOH): $\lambda=283 \mathrm{~nm}$. IR (ATR): $\nu=$ 3365, 3000, 1596, 1512, 1492, 1463, 1438, 1389, 1324, 1302, 1283, 1256, 1237, 1198, 1158, 1107, 1032, 992, 951, 832, 774, 753, 686, 649, $632 \mathrm{~cm}^{-1} .{ }^{1} \mathrm{H}$ NMR (500 MHz, $\left.\mathrm{CDCl}_{3}\right): \delta=2.31$ $(\mathrm{s}, 3 \mathrm{H}), 3.77(\mathrm{~s}, 3 \mathrm{H}), 5.62(\mathrm{br} s, 1 \mathrm{H}), 6.43-6.45(\mathrm{~m}, 1 \mathrm{H})$, 6.58-6.60 (m, $2 \mathrm{H}), 7.01-7.04(\mathrm{~m}, 2 \mathrm{H}), 7.09-7.11(\mathrm{~m}, 2 \mathrm{H})$, 7.13-7.16 (m, $1 \mathrm{H}) .{ }^{13} \mathrm{C}$ NMR and DEPT (125 MHz, $\left.\mathrm{CDCl}_{3}\right): \delta=$ $20.68\left(\mathrm{CH}_{3}\right), 55.16\left(\mathrm{CH}_{3}\right), 102.33(\mathrm{CH}), 105.43(\mathrm{CH}), 109.32$ $(\mathrm{CH}), 119.36(2 \mathrm{CH}), 129.83(2 \mathrm{CH}), 130.03(\mathrm{CH}), 131.18(\mathrm{C})$, 139.91 (C), 145.39 (C), 160.67 (C). EI-MS: $m / z(\%)=213$ (100) [M $\mathrm{M}^{+}$, 197 (4), 182 (4), 168 (4), 154 (5). HRMS: $m / z$ calcd for $\mathrm{C}_{14} \mathrm{H}_{15} \mathrm{NO}\left[\mathrm{M}^{+}\right]:$213.1154; found: 213.1158. Elemental analysis calcd for $\mathrm{C}_{14} \mathrm{H}_{15} \mathrm{NO}$ : C 78.84, $\mathrm{H}$ 7.09, N 6.57; found: $\mathrm{C} 78.97$, H 7.08, N 6.40\%.

Crystal data for 13: $\mathrm{C}_{14} \mathrm{H}_{15} \mathrm{NO}, M=213.27 \mathrm{~g} \mathrm{~mol}^{-1}$, crystal size: $0.50 \times 0.40 \times 0.10 \mathrm{~mm}^{3}$, monoclinic, space group $P 2_{1} / c$, $a=8.856(1) \AA, b=13.861(1) \AA, c=10.868(1) \AA, \beta=92.41(1)^{\circ}$, $V=1332.9(2) \AA^{3}, Z=4, \rho_{\text {calcd }}=1.063 \mathrm{~g} \mathrm{~cm}^{-3}, \mu=0.067 \mathrm{~mm}^{-1}$, $T=293(2) \mathrm{K}, \lambda=0.71073 \AA, \theta$ range $3.26-25.37^{\circ}, 18034$ reflections collected, 2165 independent reflections $\left(R_{\text {int }}=0.0280\right)$, 151 parameters. The structure was solved by direct methods and refined by full-matrix least-squares on $F^{2}$; final $R$ indices $[I>2 \sigma(I)]: R_{1}=0.0436, \mathrm{w} R_{2}=0.1205$; maximal residual electron density: 0.179 e $\AA^{-3}$ (Fig. S1

Method B: m-Anisidine (18.9 g, $153 \mathrm{mmol})$ was added portionwise over a period of $3 \mathrm{~h}$ to a solution of $p$-bromotoluene $(20.0 \mathrm{~g}, 117 \mathrm{~mol})$, caesium carbonate $(45.7 \mathrm{~g}, 140 \mathrm{mmol})$, racBINAP (3.61 g, $5.80 \mathrm{mmol})$ and palladium(II) acetate $(1.53 \mathrm{~g}$, $6.82 \mathrm{mmol})$ in toluene $(80 \mathrm{~mL})$ at reflux. The mixture was heated at reflux for $13 \mathrm{~h}$ (total reaction time $16 \mathrm{~h}$ ), then cooled to room temperature, filtered over a short pad of silica gel and Celite (diethyl ether), and the solvent was removed. Purification of the residue by column chromatography on silica gel (petroleum ether-acetone, $15: 1$ ) provided the diarylamine 13 
as light yellow solid, yield: $25.4 \mathrm{~g}$ (100\%). Spectroscopic data, see above.

2-Methoxy-6-methylcarbazole (14) and glycoborine (5methoxy-3-methylcarbazole) (15). Palladium(II) acetate (126 mg, $561 \mu \mathrm{mol}$ ) was added at $100{ }^{\circ} \mathrm{C}$ to a mixture of the diarylamine 13 (4.02 g, $18.8 \mathrm{mmol}$ ), potassium carbonate (261 $\mathrm{mg}, 1.89 \mathrm{mmol}$ ) and pivalic acid (10.2 g) in a $50 \mathrm{~mL}$ test tube. The mixture was heated and vigorously stirred at $100{ }^{\circ} \mathrm{C}$ for $20.5 \mathrm{~h}$, then cooled to room temperature, diluted with ethyl acetate and washed with a saturated solution of potassium carbonate, brine and water. The aqueous layers were extracted with ethyl acetate. The combined organic layers were dried over sodium sulfate and the solvent was evaporated. Purification of the residue by column chromatography on silica gel (pentane-dichloromethane-ethyl acetate, gradient elution, $119: 1: 0.2$ to $29: 1: 0.2)$ provided 2-methoxy-6-methylcarbazole (14) as colourless solid, yield: $3.52 \mathrm{~g}$ (89\%), m.p. 230-233 ${ }^{\circ} \mathrm{C}$ (ref. 16: $227-228{ }^{\circ} \mathrm{C}$ ). For spectroscopic data, see ref. 16. Glycoborine (5-methoxy-3-methylcarbazole) (15) was obtained from the less polar fraction as by-product in up to 5\% yield, m.p. $138-140^{\circ} \mathrm{C}$ (ref. $18 a$ : $\left.155-156^{\circ} \mathrm{C}\right)$. UV $(\mathrm{MeOH})$ : $\lambda=226,238,244,254$ (sh), 277 (sh), 287, 323, 337 mm. Fluorescence $(\mathrm{MeOH}): \lambda_{\mathrm{ex}}=287 \mathrm{~nm}, \lambda_{\mathrm{em}}=345,359 \mathrm{~nm}$. IR (ATR): $\nu=3398,3048,3012,2913,2846,1624,1604,1585,1554,1506$, $1473,1453,1438,1388,1345,1314,1294,1258,1225,1178$, 1101, 1058, 973, 915, 879, 802, 781, 745, 716, 698, 620, 590, $535 \mathrm{~cm}^{-1} .{ }^{1} \mathrm{H}$ NMR $\left(500 \mathrm{MHz}, \mathrm{CDCl}_{3}\right): \delta=2.54(\mathrm{~s}, 3 \mathrm{H}), 4.09(\mathrm{~s}$, $3 \mathrm{H}), 6.67$ (d, $J=8.0 \mathrm{~Hz}, 1 \mathrm{H}), 7.02$ (d, $J=8.0 \mathrm{~Hz}, 1 \mathrm{H}), 7.21$ (dd, $J=8.2,1.3 \mathrm{~Hz}, 1 \mathrm{H}), 7.29(\mathrm{~d}, J=8.2 \mathrm{~Hz}, 1 \mathrm{H}), 7.32(\mathrm{t}, J=8.0 \mathrm{~Hz}$, $1 \mathrm{H}), 7.94$ (br s, $1 \mathrm{H}), 8.12(\mathrm{~d}, J=0.8 \mathrm{~Hz}, 1 \mathrm{H}) .{ }^{13} \mathrm{C} \mathrm{NMR}$ and DEPT (125 MHz, $\left.\mathrm{CDCl}_{3}\right): \delta=21.43\left(\mathrm{CH}_{3}\right), 55.37\left(\mathrm{CH}_{3}\right), 100.10$ $(\mathrm{CH}), 103.48(\mathrm{CH}), 109.52(\mathrm{CH}), 112.40$ (C), 122.77 (C), 122.89 $(\mathrm{CH}), 126.16(\mathrm{CH}), 126.42(\mathrm{CH}), 128.86(\mathrm{C}), 136.82(\mathrm{C}), 141.16$ (C), 156.19 (C). EI-MS (70 eV): $m / z(\%)=211$ (100) $\left[\mathrm{M}^{+}\right]$, 196 (27), 168 (68), 167 (19). Elemental analysis calcd for $\mathrm{C}_{14} \mathrm{H}_{13} \mathrm{NO}$ : C 79.59, H 6.20, N 6.63; found: C 79.86, H 6.36, N 6.69\%.

2-Hydroxy-6-methylcarbazole (10). A $1 \mathrm{M}$ solution of boron tribromide in dichloromethane $(1.00 \mathrm{~mL}, 1.00 \mathrm{mmol})$ was added at $-78{ }^{\circ} \mathrm{C}$ to a solution of 2-methoxy-6-methylcarbazole (14) $(109 \mathrm{mg}, 516 \mu \mathrm{mol})$ in dichloromethane $(25 \mathrm{~mL})$ and the solution was stirred at $-78{ }^{\circ} \mathrm{C}$ for $30 \mathrm{~min}$. The cooling was removed and the mixture was stirred for $90 \mathrm{~min}$. Methanol $(1 \mathrm{~mL})$ was added at $0{ }^{\circ} \mathrm{C}$ and the mixture was washed with a small amount of water. The aqueous layer was extracted once with diethyl ether, the combined organic layers were dried over sodium sulfate and the solvent was evaporated. Purification of the residue by column chromatography on silica gel (petroleum ether-acetone, 2:1) provided 2-hydroxy-6-methylcarbazole (10) as colourless solid, yield: $93 \mathrm{mg}$ (91\%), m.p. $262-264{ }^{\circ} \mathrm{C}$ (ref. 5: $245^{\circ} \mathrm{C}$, decomp.). UV (MeOH): $\lambda=260$, 305, 322 (sh), 335 (sh) nm. IR (ATR): $\nu=3399,3286,2913$, 2855, 1617, 1508, 1488, 1461, 1416, 1343, 1308, 1295, 1275, 1218, 1158, 1134, 1106, 1036, 955, 937, 885, 836, 824, 805, 766, 730, 690, 652, $627 \mathrm{~cm}^{-1} .{ }^{1} \mathrm{H}$ NMR $\left(500 \mathrm{MHz}\right.$, acetone- $\left.d_{6}\right): \delta=$ $2.45(\mathrm{~s}, 3 \mathrm{H}), 6.71(\mathrm{dd}, J=8.4,2.1 \mathrm{~Hz}, 1 \mathrm{H}), 6.90(\mathrm{~d}, J=2.1 \mathrm{~Hz}$, $1 \mathrm{H}), 7.08$ (dd, $J=8.2,1.0 \mathrm{~Hz}, 1 \mathrm{H}), 7.28(\mathrm{~d}, J=8.2 \mathrm{~Hz}, 1 \mathrm{H})$, $7.74(\mathrm{~s}, 1 \mathrm{H}), 7.85$ (d, $J=8.4 \mathrm{~Hz}, 1 \mathrm{H}), 8.32$ (s, $1 \mathrm{H}), 9.94(\mathrm{br} \mathrm{s}$, $1 \mathrm{H}) .{ }^{13} \mathrm{C}$ NMR and DEPT (125 MHz, acetone- $\left.d_{6}\right): \delta=21.48\left(\mathrm{CH}_{3}\right)$, $97.34(\mathrm{CH}), 109.06(\mathrm{CH}), 110.92(\mathrm{CH}), 117.03(\mathrm{C}), 119.78(\mathrm{CH})$, $121.46(\mathrm{CH}), 124.65(\mathrm{C}), 125.99(\mathrm{CH}), 128.42$ (C), $139.17(\mathrm{C})$, 142.93 (C), 157.38 (C). EI-MS (70 eV): $m / z(\%)=197$ (100) $\left[\mathrm{M}^{+}\right]$, 196 (55), 167 (7). HRMS: $m / z$ calcd for $\mathrm{C}_{13} \mathrm{H}_{11} \mathrm{NO}\left[\mathrm{M}^{+}\right]$: 197.0841; found: 197.0855 .

Isogirinimbine (3,3,8-trimethyl-3,11-dihydropyrano[3,2-a]carbazole) (3) and 1,1,7-trimethyl-2-methylene-1,10-dihydro$2 \boldsymbol{H}$-furo[3,2-a]carbazole (17). A solution of 2-methylbut-3-yn2-ol (16) (54 $\mu \mathrm{L}, 47 \mathrm{mg}, 0.56 \mathrm{mmol})$, DBU (114 $\mu \mathrm{L}, 116 \mathrm{mg}, 762$ $\mu \mathrm{mol})$ and trifluoroacetic anhydride $(78 \mu \mathrm{L}, 0.12 \mathrm{~g}, 0.56 \mathrm{mmol})$ in acetonitrile $(2 \mathrm{~mL})$ was stirred at $-15{ }^{\circ} \mathrm{C}$ for $90 \mathrm{~min}$. The mixture was then added to a solution of 2-hydroxy-6-methylcarbazole (10) (100 mg, $507 \mu \mathrm{mol})$ and copper(I) iodide $(0.2 \mathrm{mg}$, $1 \mu \mathrm{mol})$ in acetonitrile $(4 \mathrm{~mL})$ and the mixture was stirred at $-15{ }^{\circ} \mathrm{C}$ for $40 \mathrm{~min}$. DBU $(98 \mu \mathrm{L}, 0.10 \mathrm{~g}, 0.66 \mathrm{mmol})$ was added and the mixture was stirred at $-15{ }^{\circ} \mathrm{C}$ for $3 \mathrm{~h}$ and at room temperature for $90 \mathrm{~min}$. The mixture was washed twice with water and brine and the solvent was evaporated. Toluene $(10 \mathrm{~mL})$ was added to the residue, the solution was heated at reflux for $23 \mathrm{~h}$ and the solvent was evaporated. Purification of the residue by column chromatography on silica gel (petroleum ether-dichloromethane, gradient elution, $99: 1$ to $3: 1$ ) provided isogirinimbine (3) as colourless solid, yield: $84.4 \mathrm{mg}$ (63\%), m.p. 186-187 ${ }^{\circ} \mathrm{C}$. UV (MeOH): $\lambda=221$ (sh), 237, 278 (sh), 289, 332, 337, $353 \mathrm{~nm}$. Fluorescence $(\mathrm{MeOH}): \lambda_{\text {ex }}=$ $289 \mathrm{~nm}, \lambda_{\mathrm{em}}=362,378 \mathrm{~nm}$. IR (ATR): $\nu=3415,2969,2919$, 2853, 1640, 1606, 1519, 1474, 1460, 1418, 1400, 1373, 1359, 1339, 1295, 1207, 1157, 1114, 1071, 1036, 898, 882, 859, 800, 746, 721, 704, 652, 585, 578, 562, $540 \mathrm{~cm}^{-1} \cdot{ }^{1} \mathrm{H}$ NMR (500 MHz, acetone- $\left.d_{6}\right): \delta=1.44(\mathrm{~s}, 6 \mathrm{H}), 2.45(\mathrm{~s}, 3 \mathrm{H}), 5.76(\mathrm{~d}, J$ $=9.8 \mathrm{~Hz}, 1 \mathrm{H}), 6.63(\mathrm{~d}, J=8.4 \mathrm{~Hz}, 1 \mathrm{H}), 6.90(\mathrm{~d}, J=9.8 \mathrm{~Hz}, 1 \mathrm{H})$, $7.11(\mathrm{dd}, J=8.2,1.1 \mathrm{~Hz}, 1 \mathrm{H}), 7.30(\mathrm{~d}, J=8.2 \mathrm{~Hz}, 1 \mathrm{H}), 7.76(\mathrm{~d}, J$ $=0.6 \mathrm{~Hz}, 1 \mathrm{H}), 7.79(\mathrm{~d}, J=8.4 \mathrm{~Hz}, 1 \mathrm{H}), 10.25$ (br s, $1 \mathrm{H}) .{ }^{13} \mathrm{C}$ NMR and DEPT (125 MHz, acetone- $\left.d_{6}\right): \delta=21.48\left(\mathrm{CH}_{3}\right), 27.84$ $\left(2 \mathrm{CH}_{3}\right), 76.48(\mathrm{C}), 105.66(\mathrm{C}), 109.71(\mathrm{CH}), 111.18(\mathrm{CH}), 118.18$ (C), $118.34(\mathrm{CH}), 119.98(\mathrm{CH}), 121.01(\mathrm{CH}), 124.70(\mathrm{C}), 126.42$ (CH), 128.88 (C), 129.99 (CH), 137.99 (C), 139.34 (C), 152.32

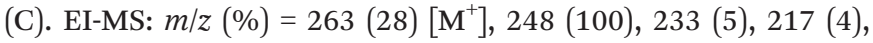
204 (9), 124 (14). HRMS: $m / z$ calcd for $\mathrm{C}_{18} \mathrm{H}_{17} \mathrm{NO}\left[\mathrm{M}^{+}\right]$: 263.1310; found: 263.1302 .

1,1,7-Trimethyl-2-methylene-1,10-dihydro- $2 H$-furo[3,2- $a]$ carbazole (17) was obtained as a by-product in up to $3 \%$ yield as colourless oil. ${ }^{1} \mathrm{H}$ NMR (300 MHz, acetone- $\left.d_{6}\right): \delta=1.68(\mathrm{~s}, 6 \mathrm{H})$, $2.46(\mathrm{~s}, 3 \mathrm{H}), 4.35$ (d, $J=2.6 \mathrm{~Hz}, 1 \mathrm{H}), 4.61(\mathrm{~d}, J=2.6 \mathrm{~Hz}, 1 \mathrm{H})$, $6.80(\mathrm{~d}, J=8.3 \mathrm{~Hz}, 1 \mathrm{H}), 7.14(\mathrm{dd}, J=8.1,1.0 \mathrm{~Hz}, 1 \mathrm{H}), 7.32(\mathrm{~d}$, $J=8.1 \mathrm{~Hz}, 1 \mathrm{H}), 7.82(\mathrm{~d}, J=1.0 \mathrm{~Hz}, 1 \mathrm{H}), 7.92$, (d, $J=8.3 \mathrm{~Hz}$, $1 \mathrm{H}), 10.21$ (br s, $1 \mathrm{H}) .{ }^{13} \mathrm{C}$ NMR and DEPT (75 MHz, acetone$\left.d_{6}\right): \delta=21.46\left(\mathrm{CH}_{3}\right), 28.69\left(2 \mathrm{CH}_{3}\right), 44.78(\mathrm{C}), 82.07\left(\mathrm{CH}_{2}\right)$, $105.52(\mathrm{CH}), 111.39(\mathrm{CH}), 116.22(\mathrm{C}), 120.02(\mathrm{CH}), 120.25(\mathrm{C})$, $120.48(\mathrm{CH}), 124.56(\mathrm{C}), 126.83(\mathrm{CH}), 129.07(\mathrm{C}), 136.56(\mathrm{C})$, 139.62 (C), 155.55 (C), 173.56 (C). ESI-MS (-25 V): $m / z=262$ $\left[(\mathrm{M}-\mathrm{H})^{-}\right]$.

Methyl 3,7-dimethyloct-6-en-1-yn-3-yl carbonate (18). A $0.5 \mathrm{M}$ solution of ethynylmagnesium bromide in THF $(51.0 \mathrm{~mL}$, 
$25.5 \mathrm{mmol}$ ) was added dropwise over a period of $15 \mathrm{~min}$ to a solution of 6-methylhept-5-en-2-one (2.94 mL, $2.52 \mathrm{~g}$, $20.0 \mathrm{mmol})$ in THF $(20 \mathrm{~mL})$ at $-78{ }^{\circ} \mathrm{C}$. The cooling was removed and the mixture was stirred for $2.5 \mathrm{~h}$. The mixture was cooled to $-78{ }^{\circ} \mathrm{C}$, methyl chloroformate $(3.06 \mathrm{~mL}, 3.74 \mathrm{~g}$, $39.6 \mathrm{mmol}$ ) was added dropwise over a period of $5 \mathrm{~min}$, the cooling was removed and the mixture was stirred for $2 \mathrm{~h}$. A saturated aqueous solution of sodium hydrogencarbonate and diethyl ether were added, and the layers were separated. The organic layer was washed with water and brine. The aqueous layers were extracted with diethyl ether, the combined organic layers were dried over sodium sulfate and the solvent was evaporated. Purification of the residue by column chromatography on silica gel (pentane-diethyl ether, gradient elution, 100:1 to $20: 1)$ provided carbonate 18 as colourless liquid, yield: $3.68 \mathrm{~g}$ (88\%). IR (ATR): $\nu=3287,2959,2923,2853,1754,1699,1684$, 1651, 1635, 1441, 1376, 1338, 1257, 1165, 1114, 1074, 1022, 940, 884, 834, 790, 666, $631 \mathrm{~cm}^{-1} \cdot{ }^{1} \mathrm{H}$ NMR (500 MHz, $\left.\mathrm{CDCl}_{3}\right): \delta=$ $1.60(\mathrm{~s}, 3 \mathrm{H}), 1.66(\mathrm{~d}, J=0.8 \mathrm{~Hz}, 3 \mathrm{H}), 1.70(\mathrm{~s}, 3 \mathrm{H}), 1.82(\mathrm{ddd}, J=$ 13.6, 11.0, $5.9 \mathrm{~Hz}, 1 \mathrm{H}$ ), 1.96 (ddd, $J=13.6,10.6,6.0 \mathrm{~Hz}, 1 \mathrm{H}$ ), 2.11-2.23 (m, $2 \mathrm{H}), 2.58$ (s, $1 \mathrm{H}), 3.75$ (s, $3 \mathrm{H}), 5.09(\mathrm{~m}, 1 \mathrm{H}) \cdot{ }^{13} \mathrm{C}$ NMR and DEPT (125 MHz, $\left.\mathrm{CDCl}_{3}\right): \delta(\mathrm{ppm})=17.57\left(\mathrm{CH}_{3}\right), 22.79$ $\left(\mathrm{CH}_{2}\right), 25.59\left(\mathrm{CH}_{3}\right), 26.17\left(\mathrm{CH}_{3}\right), 41.17\left(\mathrm{CH}_{2}\right), 54.29\left(\mathrm{CH}_{3}\right), 73.85$ $(\mathrm{CH}), 76.78$ (C), 83.03 (C), $122.85(\mathrm{CH}), 132.43(\mathrm{C}), 153.49$ $(\mathrm{C}=\mathrm{O})$. EI-MS (70 eV): $m / z(\%)=210(0.4)\left[\mathrm{M}^{+}\right], 151(7), 119$ (100), 105 (13), 91 (50), 69 (44). Elemental analysis calcd for $\mathrm{C}_{12} \mathrm{H}_{18} \mathrm{O}_{3}$ : C 68.54, $\mathrm{H}$ 8.63; found: $\mathrm{C} 68.38, \mathrm{H}$ 8.93\%.

$( \pm)$-Mahanimbicine $[( \pm)$-isomahanimbine $][( \pm)-4]$ and 1,7dimethyl-2-methylene-1-(4-methylpent-3-en-1-yl)-1,10-dihydro2H-furo[3,2-a]carbazole (19). Method A: A solution of 3,7dimethyloct-6-en-1-yn-3-yl methyl carbonate (18) (724 mg, $3.44 \mathrm{mmol})$ in acetonitrile $(11.5 \mathrm{~mL})$ was added at room temperature over a period of $8 \mathrm{~h}$ to a solution of 2-hydroxy-6-methylcarbazole (10) (453 mg, $2.30 \mathrm{mmol}$ ), DBU (0.69 mL, $0.70 \mathrm{~g}$, $4.6 \mathrm{mmol})$, and copper(I) iodide $(2.3 \mathrm{mg}, 12 \mu \mathrm{mol})$ in acetonitrile $(59 \mathrm{~mL})$. The mixture was stirred at room temperature for $14 \mathrm{~h}$ (total reaction time: $22 \mathrm{~h}$ ). Diethyl ether was added and the mixture was washed with a saturated aqueous solution of ammonium chloride and brine. The aqueous layers were extracted with diethyl ether, the combined organic layers were dried over sodium sulfate, the solvent was evaporated and the residue was dried in vacuum. The crude product was dissolved in toluene $(40 \mathrm{~mL})$, the solution was heated at reflux for $22.5 \mathrm{~h}$ and the solvent was evaporated. Purification of the residue by column chromatography on silica gel (pentane-dichloromethane-ethyl acetate, gradient elution, $294: 5: 1$ to $69: 5: 1)$ provided $10(154 \mathrm{mg}, 34 \%)$ and ( \pm )-mahanimbicine $[( \pm)-4]$ as colourless solid, yield: $372 \mathrm{mg}$ (49\%), m.p. $137-139{ }^{\circ} \mathrm{C}$ (ref. 5 and 7: $\left.142^{\circ} \mathrm{C}\right)$. UV (MeOH): $\lambda=238,289,335,353 \mathrm{~nm}$. Fluorescence $(\mathrm{MeOH}): \lambda_{\mathrm{ex}}=289 \mathrm{~nm}, \lambda_{\mathrm{em}}=361 \mathrm{~nm}$. IR (ATR): $\nu=$ 3428, 3409, 3012, 2968, 2916, 2856, 2727, 1639, 1608, 1517, 1473, 1454, 1419, 1402, 1374, 1338, 1297, 1223, 1208, 1190, 1164, 1126, 1090, 1036, 945, 909, 882, 816, 798, 748, 719, 673, $584 \mathrm{~cm}^{-1} .{ }^{1} \mathrm{H}$ NMR $\left(500 \mathrm{MHz}, \mathrm{CDCl}_{3}\right): \delta=1.45(\mathrm{~s}, 3 \mathrm{H}), 1.58(\mathrm{~s}$, $3 \mathrm{H}), 1.66(\mathrm{~d}, J=0.8 \mathrm{~Hz}, 3 \mathrm{H}), 1.70-1.82(\mathrm{~m}, 2 \mathrm{H}), 2.11-2.21(\mathrm{~m}$, $2 \mathrm{H}), 2.50(\mathrm{~s}, 3 \mathrm{H}), 5.11(\mathrm{~m}, 1 \mathrm{H}), 5.66(\mathrm{~d}, J=9.8 \mathrm{~Hz}, 1 \mathrm{H})$, $6.64(\mathrm{~d}, J=9.8 \mathrm{~Hz}, 1 \mathrm{H}), 6.72(\mathrm{dd}, J=8.3,0.5 \mathrm{~Hz}, 1 \mathrm{H}), 7.14(\mathrm{dd}$, $J=8.2,1.1 \mathrm{~Hz}, 1 \mathrm{H}), 7.28(\mathrm{~d}, J=8.2 \mathrm{~Hz}, 1 \mathrm{H}), 7.73(\mathrm{~s}, 1 \mathrm{H}), 7.74$ $(\mathrm{d}, J=8.3 \mathrm{~Hz}, 1 \mathrm{H}), 7.84$ (br s, $1 \mathrm{H}) .{ }^{13} \mathrm{C}$ NMR and DEPT $\left(125 \mathrm{MHz}, \mathrm{CDCl}_{3}\right): \delta=17.63\left(\mathrm{CH}_{3}\right), 21.44\left(\mathrm{CH}_{3}\right), 22.73\left(\mathrm{CH}_{2}\right)$, $25.66\left(\mathrm{CH}_{3}\right), 25.95\left(\mathrm{CH}_{3}\right), 40.80\left(\mathrm{CH}_{2}\right), 78.30(\mathrm{C}), 104.51(\mathrm{C})$, $109.44(\mathrm{CH}), 110.06(\mathrm{CH}), 117.30(\mathrm{CH}), 117.39(\mathrm{C}), 119.45(\mathrm{CH})$, $120.39(\mathrm{CH}), 124.11(\mathrm{CH}), 124.14(\mathrm{C}), 125.73(\mathrm{CH}), 128.65(\mathrm{CH})$, 129.01 (C), 131.71 (C), 136.56 (C), 137.70 (C), 151.70 (C). EI-MS $(70 \mathrm{eV}): m / z(\%)=331(45)\left[\mathrm{M}^{+}\right], 288$ (11), 248 (100), (10), 210 (51), 209 (36), 180 (17). Elemental analysis calcd for $\mathrm{C}_{23} \mathrm{H}_{25} \mathrm{NO}$ : C 83.34, H 7.60, N 4.23; found: C 83.59, H 7.88, N 4.23\%.

The furo[3,2- $a$ ]carbazole 19 was obtained as by-product in up to $5 \%$ yield. ${ }^{1} \mathrm{H}$ NMR (500 $\left.\mathrm{MHz}, \mathrm{CDCl}_{3}\right): \delta=1.35(\mathrm{~s}, 3 \mathrm{H})$, 1.47-1.53 (m, $1 \mathrm{H}), 1.53$ (s, $3 \mathrm{H}), 1.64$ (s, $3 \mathrm{H}), 1.78$ (ddd, $J=$ 13.4, 11.9, $4.6 \mathrm{~Hz}, 1 \mathrm{H}$ ), 1.90-1.98 (m, $1 \mathrm{H}$ ), 2.12 (ddd, $J=13.4$, 11.9, $4.8 \mathrm{~Hz}, 1 \mathrm{H}), 2.51$ (s, $3 \mathrm{H}), 4.24$ (d, $J=2.8 \mathrm{~Hz}, 1 \mathrm{H}), 4.77$ $(\mathrm{d}, J=2.8 \mathrm{~Hz}, 1 \mathrm{H}), 4.97(\mathrm{~m}, 1 \mathrm{H}), 6.84(\mathrm{~d}, J=8.3 \mathrm{~Hz}, 1 \mathrm{H}), 7.17$ $(\mathrm{dd}, J=8.2,1.0 \mathrm{~Hz}, 1 \mathrm{H}), 7.30(\mathrm{~d}, J=8.2 \mathrm{~Hz}, 1 \mathrm{H}), 7.74(\mathrm{~m}$, $1 \mathrm{H}), 7.77$ (d, $J=1.0 \mathrm{~Hz}, 1 \mathrm{H}), 7.83$ (d, $J=8.3 \mathrm{~Hz}, 1 \mathrm{H}) .{ }^{13} \mathrm{C} \mathrm{NMR}$ and DEPT (125 MHz, $\left.\mathrm{CDCl}_{3}\right): \delta(\mathrm{ppm})=17.61\left(\mathrm{CH}_{3}\right), 21.58$ $\left(\mathrm{CH}_{3}\right), 24.21\left(\mathrm{CH}_{2}\right), 25.70\left(\mathrm{CH}_{3}\right), 28.65\left(\mathrm{CH}_{3}\right), 41.95\left(\mathrm{CH}_{2}\right)$, $48.39(\mathrm{C}), 82.67\left(\mathrm{CH}_{2}\right), 102.61(\mathrm{CH}), 110.29(\mathrm{CH}), 113.24(\mathrm{C})$, $119.47(\mathrm{C}), 119.67(\mathrm{CH}), 119.99(\mathrm{CH}), 123.52(\mathrm{CH}), 124.10(\mathrm{C})$, 126.29 (CH), 129.40 (C), 132.30 (C), 135.74 (C), 137.95 (C), 155.63 (C), $170.22(\mathrm{C})$.

2-Methoxy-6-methyl-9-tosylcarbazole (20). Sodium hydride (539 $\mathrm{mg}$ of a $60 \%$ dispersion in mineral oil, $13.5 \mathrm{mmol}$ ) was added at $0{ }^{\circ} \mathrm{C}$ to a solution of 2-methoxy-6-methylcarbazole (14) (701 $\mathrm{mg}, 3.32 \mathrm{mmol})$ in THF (35 mL) and the mixture was stirred at $0{ }^{\circ} \mathrm{C}$ for $1.25 \mathrm{~h}$. $p$-Toluenesulfonyl chloride $(949 \mathrm{mg}$, $4.98 \mathrm{mmol}$ ) was added at $0{ }^{\circ} \mathrm{C}$, the cooling was removed and the mixture was stirred for $16.25 \mathrm{~h}$. The mixture was diluted with diethyl ether and washed with water and brine. The aqueous layers were extracted with diethyl ether, the combined organic layers were dried over sodium sulfate and the solvent was evaporated. Purification of the residue by flash chromatography on silica gel (pentane-dichloromethane-ethyl, $70: 5: 1$ ) provided 2-methoxy-6-methyl-9-tosylcarbazole (20) as colourless solid, yield: $969 \mathrm{mg}$ (80\%), m.p. $145-148^{\circ} \mathrm{C}$. UV (MeOH): $\lambda$ $=224,268,296,309,326 \mathrm{~nm}$. Fluorescence $(\mathrm{MeOH}): \lambda_{\mathrm{ex}}=$ $268 \mathrm{~nm}, \lambda_{\mathrm{em}}=396 \mathrm{~nm}$. IR (ATR): $\nu=3091,2992,2949,2917$, 2831, 1622, 1580, 1493, 1477, 1453, 1361, 1299, 1280, 1253, 1190, 1167, 1147, 1130, 1090, 1044, 985, 938, 869, 842, 801, 777, 736, 705, $675 \mathrm{~cm}^{-1} \cdot{ }^{1} \mathrm{H}$ NMR (500 MHz, acetone- $\left.d_{6}\right): \delta=$ $2.27(\mathrm{~s}, 3 \mathrm{H}), 2.43(\mathrm{~s}, 3 \mathrm{H}), 3.95(\mathrm{~s}, 3 \mathrm{H}), 7.01(\mathrm{dd}, J=8.6$, $2.3 \mathrm{~Hz}, 1 \mathrm{H}), 7.25-7.29$ (m, $3 \mathrm{H}), 7.73-7.77$ (m, $3 \mathrm{H}), 7.86$ (d, $J=$ $2.3 \mathrm{~Hz}, 1 \mathrm{H}), 7.90$ (d, $J=8.6 \mathrm{~Hz}, 1 \mathrm{H}), 8.14$ (d, $J=8.5 \mathrm{~Hz}, 1 \mathrm{H})$. ${ }^{13} \mathrm{C}$ NMR and DEPT (125 MHz, acetone- $\left.d_{6}\right): \delta=21.21\left(\mathrm{CH}_{3}\right)$, $21.35\left(\mathrm{CH}_{3}\right), 56.07\left(\mathrm{CH}_{3}\right), 100.72(\mathrm{CH}), 112.87(\mathrm{CH}), 115.55$ $(\mathrm{CH}), 120.43(\mathrm{CH}), 120.60(\mathrm{C}), 121.76(\mathrm{CH}), 127.38(2 \mathrm{CH})$, $127.63(\mathrm{C}), 128.10(\mathrm{CH}), 130.73(2 \mathrm{CH}), 134.78(\mathrm{C}), 135.54(\mathrm{C})$, 137.25 (C), 140.76 (C), 146.38 (C), 160.86 (C). EI-MS (70 eV): $\mathrm{m} / z(\%)=365(65)\left[\mathrm{M}^{+}\right], 210$ (100), 167 (26). HRMS: $\mathrm{m} / z$ calcd for $\mathrm{C}_{21} \mathrm{H}_{19} \mathrm{NO}_{3} \mathrm{~S}\left[\mathrm{M}^{+}\right]$: 365.1086; found: 365.1099. Elemental analysis calcd for $\mathrm{C}_{21} \mathrm{H}_{19} \mathrm{NO}_{3} \mathrm{~S}$ : C 69.02, H 5.24, N 3.83, S 8.77; found: C 69.14, H 5.20, N 3.82, S 9.31\%. 
$\mathrm{N}$-Tosylmahanimbicine (21). A $1 \mathrm{M}$ solution of boron tribromide in dichloromethane $(4.1 \mathrm{~mL}, 4.1 \mathrm{mmol})$ was added dropwise at $-78^{\circ} \mathrm{C}$ to a solution of 2-methoxy-6-methyl-9-tosylcarbazole (20) (502 mg, $1.37 \mathrm{mmol}$ ). The cooling was removed and the mixture was stirred for $2.5 \mathrm{~h}$. Methanol $(4 \mathrm{~mL})$ was added, the mixture was diluted with diethyl ether, and washed with water and brine. The aqueous layer was extracted with diethyl ether, the combined organic layers were dried over sodium sulfate and the solvent was evaporated. Drying in vacuum provided crude 2-hydroxy-6-methyl-9-tosylcarbazole (539 mg) as light yellow solid, m.p. $166-168^{\circ} \mathrm{C}$.

2-Hydroxy-6-methyl-9-tosylcarbazole: UV (MeOH): $\lambda=222$, 244 (sh), 260, 267, 275, 297, $308 \mathrm{~nm}$. Fluorescence $(\mathrm{MeOH}): \lambda_{\text {ex }}$ $=260 \mathrm{~nm}, \lambda_{\mathrm{em}}=381 \mathrm{~nm}$. IR (ATR): $\nu=3482,3035,2919,1612$, 1558, 1499, 1442, 1398, 1346, 1296, 1268, 1215, 1186, 1161, 1114, 1088, 1027, 991, 952, 854, 811, 790, 739, 718, 701, 679, $660,604 \mathrm{~cm}^{-1} \cdot{ }^{1} \mathrm{H}$ NMR $\left(500 \mathrm{MHz}\right.$, acetone- $\left.d_{6}\right): \delta=2.28(\mathrm{~s}$, $3 \mathrm{H}), 2.42(\mathrm{~s}, 3 \mathrm{H}), 6.92(\mathrm{dd}, J=8.5,2.1 \mathrm{~Hz}, 1 \mathrm{H}), 7.23-7.27(\mathrm{~m}$, $3 \mathrm{H}), 7.69-7.74(\mathrm{~m}, 3 \mathrm{H}), 7.81(\mathrm{~d}, J=2.4 \mathrm{~Hz}, 1 \mathrm{H}), 7.82(\mathrm{~d}, J=$ $8.2 \mathrm{~Hz}, 1 \mathrm{H}), 8.11$ (d, $J=8.5 \mathrm{~Hz}, 1 \mathrm{H}), 8.80(\mathrm{~s}, 1 \mathrm{H}) .{ }^{13} \mathrm{C}$ NMR and DEPT (125 MHz, acetone- $\left.d_{6}\right): \delta=21.17\left(\mathrm{CH}_{3}\right), 21.32\left(\mathrm{CH}_{3}\right)$, $102.55(\mathrm{CH}), 113.72(\mathrm{CH}), 115.44(\mathrm{CH}), 119.70(\mathrm{C}), 120.16(\mathrm{CH})$, $121.77(\mathrm{CH}), 127.28(2 \mathrm{CH}), 127.72(\mathrm{CH}), 127.87(\mathrm{C}), 130.66$ (2 CH), 134.62 (C), 135.64 (C), 137.09 (C), 140.90 (C), 146.26 (C), 158.55 (C). EI-MS (70 eV): $m / z(\%)=351$ (79) $\left[\mathrm{M}^{+}\right], 196$ (100), 167 (12), 91 (5). HRMS: $m / z$ calcd for $\mathrm{C}_{20} \mathrm{H}_{17} \mathrm{NO}_{3} \mathrm{~S}\left[\mathrm{M}^{+}\right]$: 351.0929; found: 351.0926. Elemental analysis calcd for $\mathrm{C}_{20} \mathrm{H}_{17} \mathrm{NO}_{3} \mathrm{~S}$ : C 68.36, $\mathrm{H} 4.88, \mathrm{~N} 3.99$, $\mathrm{S} 9.12$; found: $\mathrm{C} 68.55, \mathrm{H}$ 4.86, N 3.94, S 9.78\%.

A solution of 3,7-dimethyloct-6-en-1-yn-3-yl methyl carbonate $(18)(577 \mathrm{mg}, 2.74 \mathrm{mmol})$ in acetonitrile $(12 \mathrm{~mL})$ was added over a period of $12 \mathrm{~h}$ at room temperature to a solution of the crude 2-hydroxy-6-methyl-9-tosylcarbazole (539 mg), DBU (0.62 mL, $0.63 \mathrm{~g}, 4.1 \mathrm{mmol})$ and copper(I) iodide $(1.3 \mathrm{mg}$, $7 \mu \mathrm{mol})$ in acetonitrile $(35 \mathrm{~mL})$ and the mixture was stirred at room temperature for $10 \mathrm{~h}$ (total reaction time $22 \mathrm{~h}$ ). The mixture was diluted with diethyl ether and washed with water, $10 \%$ aqueous $\mathrm{HCl}$ and brine. The aqueous layers were extracted with diethyl ether, the combined organic layers were dried over sodium sulfate, the solvent was evaporated and the residue was dried in vacuum. The crude product was dissolved in xylenes $(25 \mathrm{~mL})$, the solution was heated at reflux for $27.5 \mathrm{~h}$ and the solvent was evaporated. Purification of the residue by column chromatography on silica gel (pentane-dichloromethane-ethyl acetate, gradient elution, 99:1 to $15: 1$ ) provided a mixture of $N$-tosylmahanimbicine (21) and compound 22, combined yield: $546 \mathrm{mg}$ (82\%), ratio of $21: 22=7.7: 1$ (determined by ${ }^{1} \mathrm{H}$ NMR integration). UV (MeOH): $\lambda=230,266$, $313 \mathrm{~nm}$. Fluorescence $(\mathrm{MeOH}): \lambda_{\mathrm{ex}}=266 \mathrm{~nm}, \lambda_{\mathrm{em}}=359$, $419 \mathrm{~nm}$. IR (ATR): $\nu=2967,2921,2853,1725,1631,1596,1476$, 1451, 1398, 1368, 1272, 1172, 1086, 1035, 960, 918, 890, 810, $782,749,726,703,672,662,629,575,542 \mathrm{~cm}^{-1} \cdot{ }^{1} \mathrm{H} \mathrm{NMR}$ $\left(500 \mathrm{MHz}\right.$, acetone $\left.-d_{6}\right): \delta$ (major isomer) $=1.52(\mathrm{~s}, 3 \mathrm{H}), 1.58(\mathrm{~s}$, $3 \mathrm{H}), 1.67$ (s, $3 \mathrm{H}), 1.80-1.90$ (m, $2 \mathrm{H}), 2.18-2.24$ (m, $2 \mathrm{H}), 2.21$ $(\mathrm{s}, 3 \mathrm{H}), 2.39(\mathrm{~s}, 3 \mathrm{H}), 5.18(\mathrm{~m}, 1 \mathrm{H}), 5.80(\mathrm{~d}, J=10.0 \mathrm{~Hz}, 1 \mathrm{H})$, 6.88 (dd, $J=8.5,0.7 \mathrm{~Hz}, 1 \mathrm{H}), 7.00$ (d, $J=8.2 \mathrm{~Hz}, 2 \mathrm{H}), 7.08$ (m,
$2 \mathrm{H}), 7.24(\mathrm{~d}, J=10.0 \mathrm{~Hz}, 1 \mathrm{H}), 7.24(\mathrm{~m}, 1 \mathrm{H}), 7.52(\mathrm{~d}, J=0.7$ $\mathrm{Hz}, 1 \mathrm{H}), 7.61$ (d, $J=8.3 \mathrm{~Hz}, 1 \mathrm{H}), 8.04$ (d, $J=8.3 \mathrm{~Hz}, 1 \mathrm{H}) .{ }^{13} \mathrm{C}$ NMR and DEPT (125 MHz, acetone- $\left.d_{6}\right)$ : $\delta$ (major isomer) $=$ $17.66\left(\mathrm{CH}_{3}\right), 21.23\left(\mathrm{CH}_{3}\right), 21.27\left(\mathrm{CH}_{3}\right), 23.37\left(\mathrm{CH}_{2}\right), 25.83$ $\left(2 \mathrm{CH}_{3}\right), 40.99\left(\mathrm{CH}_{2}\right), 78.40(\mathrm{C}), 115.19(\mathrm{C}), 115.89(\mathrm{CH}), 119.61$ $(\mathrm{CH}), 120.02(\mathrm{CH}), 121.02(\mathrm{CH}), 122.80(\mathrm{CH}), 124.49(\mathrm{C}), 124.97$ $(\mathrm{CH}), 127.53(\mathrm{CH}), 127.82(2 \mathrm{CH}), 127.83(\mathrm{CH}), 129.73(2 \mathrm{CH})$, 130.55 (C), 132.08 (C), 133.15 (C), 136.34 (C), 138.19 (C), 139.75 (C), 145.67 (C), 154.82 (C). ESI-MS (+25 V): $\mathrm{m} / z(\%)=486.3$ $\left[(\mathrm{M}+\mathrm{H})^{+}\right], 988.7\left[\left(2 \mathrm{M}+\mathrm{NH}_{4}\right)^{+}\right]$. Elemental analysis calcd for $\mathrm{C}_{30} \mathrm{H}_{31} \mathrm{NO}_{3} \mathrm{~S}$ : C 74.20, H 6.43, N 2.88, S 6.60; found: C 74.46, H 6.68, N 2.97, S 6.49\%.

( \pm -Mahanimbicine $[( \pm)$-isomahanimbine $][( \pm)-4]$ and $2,10-$ dihydro-2,7-dimethyl-2-(4-methylpent-3-enyl)pyrano[2,3-b]carbazole (23). Method B: A $1 \mathrm{M}$ solution of TBAF in THF $(0.28 \mathrm{~mL}$, $0.28 \mathrm{mmol}$ ) was added at room temperature to a mixture of the carbazoles 21 and $22(68.7 \mathrm{mg}, 141 \mu \mathrm{mol}$, ratio $21: 22=7.7: 1$, see above). The mixture was irradiated in the microwave reactor at $300 \mathrm{~W}$ and $70{ }^{\circ} \mathrm{C}$ for $3 \mathrm{~h}$ and then at $75{ }^{\circ} \mathrm{C}$ for $2 \mathrm{~h}$. A $1 \mathrm{M}$ solution of TBAF in THF $(0.28 \mathrm{~mL}, 0.28 \mathrm{mmol})$ was added and the mixture was irradiated in the microwave reactor at $300 \mathrm{~W}$ and $75^{\circ} \mathrm{C}$ for $1 \mathrm{~h}$. The mixture was diluted with diethyl ether, and washed first with an aqueous solution of ammonium chloride and then with brine. The aqueous layers were extracted with diethyl ether, the combined organic layers were dried over sodium sulfate and the solvent was evaporated. Purification of the residue by column chromatography on silica gel (pentane-dichloromethane-diethyl ether, gradient elution, $119: 1: 0.2$ to $14: 1: 0.2)$ provided $( \pm)$-mahanimbicine $[( \pm)-4]$ as colourless solid (yield: $37.1 \mathrm{mg}, 79 \%$; spectroscopic data, see above) and 23 as light yellow solid, yield: $4.0 \mathrm{mg}$ (9\%). ${ }^{1} \mathrm{H}-\mathrm{NMR}\left(500 \mathrm{MHz}, \mathrm{CDCl}_{3}\right): \delta=1.43$ (s, $3 \mathrm{H}$ ), 1.57 (s, 3 H), 1.65 (s, 3 H), 1.67-1.80 (m, 2 H), 2.14-2.17 (m, 2 H), 2.49 (s, $3 \mathrm{H}), 5.10$ (br t, $J=7.1 \mathrm{~Hz}, 1 \mathrm{H}), 5.55(\mathrm{~d}, J=9.8 \mathrm{~Hz}, 1 \mathrm{H})$, $6.53(\mathrm{~d}, J=9.8 \mathrm{~Hz}, 1 \mathrm{H}), 6.78(\mathrm{~s}, 1 \mathrm{H}), 7.12(\mathrm{dd}, J=8.1,1.1 \mathrm{~Hz}$, $1 \mathrm{H}), 7.23(\mathrm{~d}, J=8.1 \mathrm{~Hz}, 1 \mathrm{H}), 7.57$ (s, $1 \mathrm{H}), 7.71$ (br s, $1 \mathrm{H})$, 7.82 (br s, $1 \mathrm{H}$ ).

Murrayamine-J (5). DDQ (109 mg, $480 \mu \mathrm{mol})$ was added at room temperature to a solution of $( \pm)$-mahanimbicine $[( \pm)-4]$ (39.7 $\mathrm{mg}, 120 \mu \mathrm{mol})$ in methanol $(20 \mathrm{~mL})$, THF $(2 \mathrm{~mL})$ and water $(2 \mathrm{~mL})$. The mixture was stirred at room temperature for $2 \mathrm{~h}$. DDQ (54.4 mg, $240 \mu \mathrm{mol})$ was added, the mixture was stirred for $1.5 \mathrm{~h}$, an additional portion of DDQ (54.4 mg, 240 $\mu \mathrm{mol})$ was added and the mixture was stirred at room temperature for $2.75 \mathrm{~h}$ (total reaction time: $6.25 \mathrm{~h}$ ). The mixture was diluted with diethyl ether and washed with $2 \mathrm{~N}$ aqueous $\mathrm{NaOH}$, water and brine. The aqueous layers were extracted with diethyl ether, the combined organic layers were dried over sodium sulfate and the solvent was evaporated. Purification of the residue by column chromatography on silica gel (pentane-dichloromethane-ethyl acetate, gradient elution, $1: 0: 0$ to $13: 5: 1$ ) provided murrayamine-J (5) as colourless solid, yield: $27.9 \mathrm{mg}$ (67\%), m.p. 93-97 ${ }^{\circ} \mathrm{C}$ (ref. 8: oil). UV (MeOH): $\lambda=246,267,311,347$ (sh) nm. Fluorescence $(\mathrm{MeOH})$ : $\lambda_{\mathrm{ex}}=311 \mathrm{~nm}, \lambda_{\mathrm{em}}=350 \mathrm{~nm}$. IR (ATR): $\nu=3322,3045,2966$, 2918, 2848, 2757, 1771, 1735, 1717, 1698, 1662, 1604, 1580, 
1508, 1474, 1462, 1415, 1393, 1343, 1320, 1278, 1224, 1176, 1121, 1085, 1031, 962, 895, 813, 784, 754, 721, 662, $629 \mathrm{~cm}^{-1}$. ${ }^{1} \mathrm{H}$ NMR $\left(600 \mathrm{MHz}, \mathrm{CDCl}_{3}\right): \delta=1.46(\mathrm{~s}, 3 \mathrm{H}), 1.58(\mathrm{~s}, 3 \mathrm{H}), 1.66$ (s, $3 \mathrm{H}), 1.71-1.83(\mathrm{~m}, 2 \mathrm{H}), 2.11-2.21(\mathrm{~m}, 2 \mathrm{H}), 5.10(\mathrm{~m}, 1 \mathrm{H})$, $5.71(\mathrm{~d}, J=9.8 \mathrm{~Hz}, 1 \mathrm{H}), 6.67(\mathrm{~d}, J=9.8 \mathrm{~Hz}, 1 \mathrm{H}), 6.81(\mathrm{~d}, J=8.4$ $\mathrm{Hz}, 1 \mathrm{H}), 7.47$ (d, J = 8.4 Hz, $1 \mathrm{H}), 7.83$ (d, J=8.4 Hz, $1 \mathrm{H}), 7.89$ (dd, $J=8.4,1.4 \mathrm{~Hz}, 1 \mathrm{H}$ ), 8.35 (br s, $1 \mathrm{H}$ ), 8.46 (s, $1 \mathrm{H}$ ), 10.07 (s, $1 \mathrm{H}) .{ }^{13} \mathrm{C}$ NMR and DEPT (150 MHz, $\left.\mathrm{CDCl}_{3}\right): \delta=17.62\left(\mathrm{CH}_{3}\right)$, $22.70\left(\mathrm{CH}_{2}\right), 25.65\left(\mathrm{CH}_{3}\right), 26.05\left(\mathrm{CH}_{3}\right), 40.88\left(\mathrm{CH}_{2}\right), 78.69(\mathrm{C})$, 104.98 (C), 110.69 (CH), $110.91(\mathrm{CH}), 116.75(\mathrm{CH}), 117.07(\mathrm{C})$, $120.85(\mathrm{CH}), 122.77(\mathrm{CH}), 123.93(\mathrm{CH}), 124.23(\mathrm{C}), 126.33(\mathrm{CH})$, 129.35 (C), 129.43 (CH), 131.85 (C), 136.75 (C), 143.42 (C), 152.65 (C), 192.01 (CHO). EI-MS (70 eV): $m / z(\%)=345$ (33) $\left[\mathrm{M}^{+}\right], 330$ (4), 262 (100), 234 (4). HRMS: $\mathrm{m} / z$ calcd for $\mathrm{C}_{23} \mathrm{H}_{23} \mathrm{NO}_{2}\left[\mathrm{M}^{+}\right]: 345.1729$; found: 345.1731. Elemental analysis calcd for $\mathrm{C}_{23} \mathrm{H}_{23} \mathrm{NO}_{2}$ : $\mathrm{C}$ 79.97, $\mathrm{H}$ 6.71, $\mathrm{N}$ 4.05; found: C 79.22, H 6.94, N 4.14\%.

Bicyclomahanimbicine (6). A solution of $( \pm)$-mahanimbicine $[( \pm)-4](40.1 \mathrm{mg}, 121 \mu \mathrm{mol})$ in toluene $(40 \mathrm{~mL})$ was placed in a water bath and irradiated using a daylight lamp $(600 \mathrm{~lm}$, $6500 \mathrm{~K}$ ) for $14 \mathrm{~d}$ under continuous stirring at room temperature. Evaporation of the solvent and purification of the residue by column chromatography on silica gel (pentane-dichloromethane-ethyl acetate, gradient elution, 120:1:0.2 to $23: 1: 0.2)$ provided bicyclomahanimbicine (6) as colourless solid, yield: $15.7 \mathrm{mg}$ (39\%), m.p. $224-225{ }^{\circ} \mathrm{C}$ (ref. 5: $218{ }^{\circ} \mathrm{C}$, decomp.). UV (MeOH): $\lambda=223,242,256,261,288$ (sh), 304, 322 (sh), 335 (sh) nm. Fluorescence $(\mathrm{MeOH}): \lambda_{\mathrm{ex}}=242 \mathrm{~nm}, \lambda_{\mathrm{em}}$ $=344,359 \mathrm{~nm}$. IR (ATR): $\nu=3452,3021,2964,2944,2913$, 2856, 1711, 1609, 1508, 1483, 1454, 1415, 1381, 1365, 1339, 1293, 1217, 1194, 1177, 1142, 1085, 1022, 976, 918, 873, 802, 766, 740, 672, 645, $589 \mathrm{~cm}^{-1} \cdot{ }^{1} \mathrm{H}$ NMR (500 MHz, $\left.\mathrm{CDCl}_{3}\right): \delta=$ $0.77(\mathrm{~s}, 3 \mathrm{H}), 1.45(\mathrm{~s}, 3 \mathrm{H}), 1.57(\mathrm{~s}, 3 \mathrm{H}), 1.63-1.76(\mathrm{~m}, 3 \mathrm{H})$, 2.07-2.14 (m, $1 \mathrm{H}), 2.49-2.54(\mathrm{~m}, 1 \mathrm{H}), 2.50$ (s, $3 \mathrm{H}), 2.72(\mathrm{dd}, J=$ 9.4, 7.7 Hz, $1 \mathrm{H}), 3.28$ (d, $J=9.4 \mathrm{~Hz}, 1 \mathrm{H}), 6.77(\mathrm{~d}, J=8.4 \mathrm{~Hz}, 1$ H), $7.14(\mathrm{dd}, J=8.2,1.2 \mathrm{~Hz}, 1 \mathrm{H}), 7.28(\mathrm{~d}, J=8.2 \mathrm{~Hz}, 1 \mathrm{H}), 7.40$ (br s, $1 \mathrm{H}), 7.75(\mathrm{~s}, 1 \mathrm{H}), 7.76(\mathrm{~d}, J=8.4 \mathrm{~Hz}, 1 \mathrm{H}) .{ }^{13} \mathrm{C} \mathrm{NMR}$ and DEPT (125 MHz, $\left.\mathrm{CDCl}_{3}\right): \delta=18.53\left(\mathrm{CH}_{3}\right), 21.42\left(\mathrm{CH}_{3}\right), 25.61$ $\left(\mathrm{CH}_{2}\right), 27.57\left(\mathrm{CH}_{3}\right), 35.08\left(\mathrm{CH}_{3}\right), 37.12(\mathrm{CH}), 37.65(\mathrm{CH}), 38.00$ $\left(\mathrm{CH}_{2}\right), 39.22(\mathrm{C}), 46.39(\mathrm{CH}), 83.54(\mathrm{C}), 106.48(\mathrm{C}), 109.99(\mathrm{CH})$, $111.14(\mathrm{CH}), 116.62(\mathrm{C}), 118.94(\mathrm{CH}), 119.46(\mathrm{CH}), 124.35(\mathrm{C})$, 125.41 (CH), 128.88 (C), 137.46 (C), 139.64 (C), 151.85 (C). EI-MS $(70 \mathrm{eV}): m / z(\%)=331(30)\left[\mathrm{M}^{+}\right], 248$ (100), 234 (3), 218 (2). HRMS: $m / z$ calcd for $\mathrm{C}_{23} \mathrm{H}_{25} \mathrm{NO}\left[\mathrm{M}^{+}\right]$: 331.1936; found: 331.1937.

Murrayamine-M (7). DDQ (59 mg, $260 \mu \mathrm{mol}$ ) was added at $0{ }^{\circ} \mathrm{C}$ to a solution of bicyclomahanimbicine (6) $(28.3 \mathrm{mg}$, $85.4 \mu \mathrm{mol})$ in methanol $(10 \mathrm{~mL})$, THF $(1 \mathrm{~mL})$ and water $(2 \mathrm{~mL})$. The cooling was removed and the mixture was stirred for $2 \mathrm{~h}$ at room temperature. DDQ (38.6 $\mathrm{mg}, 0.17 \mathrm{mmol}$ ) was added, the mixture was stirred for $1.5 \mathrm{~h}$, an additional portion of DDQ (29 $\mathrm{mg}, 130 \mu \mathrm{mol}$ ) was added and the mixture was stirred for $1.5 \mathrm{~h}$ (total reaction time: $5 \mathrm{~h}$ ). The mixture was diluted with diethyl ether and washed with $2 \mathrm{~N}$ aqueous $\mathrm{NaOH}$, water and brine. The aqueous layers were extracted with diethyl ether, the combined organic layers were dried over sodium sulfate and the solvent was evaporated. Purification of the residue by column chromatography on silica gel (pentane-dichloromethane-ethyl acetate, gradient elution, $1: 0: 0$ to $14: 5: 1$ ) provided murrayamine-M (7) as light yellow solid, yield: $15 \mathrm{mg}$ (51\%), m.p. $216-218{ }^{\circ} \mathrm{C}$ (ref. 8: oil). UV (MeOH): $\lambda=243,255$ (sh), 293, 329 (sh) nm. Fluorescence $(\mathrm{MeOH}): \lambda_{\mathrm{ex}}=293 \mathrm{~nm}, \lambda_{\mathrm{em}}$ $=368 \mathrm{~nm}$. IR (ATR): $\nu=3416,3385,2947,2863$, 1735, 1698, 1673, 1654, 1605, 1572, 1508, 1474, 1458, 1414, 1318, 1220, 1182, 1151, 1114, 1088, 1021, 926, 891, 818, 787, 729, 686, 632, $610 \mathrm{~cm}^{-1} \cdot{ }^{1} \mathrm{H}$ NMR $\left(500 \mathrm{MHz}, \mathrm{CDCl}_{3}\right): \delta=0.78(\mathrm{~s}, 3 \mathrm{H}), 1.45(\mathrm{~s}$, $3 \mathrm{H}), 1.59$ (s, $3 \mathrm{H}), 1.66-1.78(\mathrm{~m}, 3 \mathrm{H}), 2.04-2.12(\mathrm{~m}, 1 \mathrm{H}), 2.54$ $(\mathrm{m}, 1 \mathrm{H}), 2.74(\mathrm{dd}, J=9.5,7.7 \mathrm{~Hz}, 1 \mathrm{H}), 3.30(\mathrm{~d}, J=9.5 \mathrm{~Hz}$, $1 \mathrm{H}), 6.88(\mathrm{~d}, J=8.5 \mathrm{~Hz}, 1 \mathrm{H}), 7.48(\mathrm{~d}, J=8.4 \mathrm{~Hz}, 1 \mathrm{H}), 7.82(\mathrm{br}$ s, $1 \mathrm{H}), 7.85(\mathrm{~d}, J=8.5 \mathrm{~Hz}, 1 \mathrm{H}), 7.88(\mathrm{dd}, J=8.4,1.6 \mathrm{~Hz}, 1 \mathrm{H})$, $8.48(\mathrm{~m}, 1 \mathrm{H}), 10.07$ (s, $1 \mathrm{H}) .{ }^{13} \mathrm{C}$ NMR and DEPT $(125 \mathrm{MHz}$, $\left.\mathrm{CDCl}_{3}\right): \delta=18.72\left(\mathrm{CH}_{3}\right), 25.59\left(\mathrm{CH}_{2}\right), 27.42\left(\mathrm{CH}_{3}\right), 35.10\left(\mathrm{CH}_{3}\right)$, $36.96(\mathrm{CH}), 37.66(\mathrm{CH}), 38.19\left(\mathrm{CH}_{2}\right), 39.30(\mathrm{C}), 46.40(\mathrm{CH})$, $83.88(\mathrm{C}), 107.17(\mathrm{C}), 110.68(\mathrm{CH}), 112.77(\mathrm{CH}), 116.48(\mathrm{C})$, $119.44(\mathrm{CH}), 122.88(\mathrm{CH}), 124.46(\mathrm{C}), 126.01(\mathrm{CH}), 129.32(\mathrm{C})$, 139.84 (C), 143.10 (C), 152.92 (C), 192.03 (CHO). EI-MS (70 eV): $m / z(\%)=345(24)\left[\mathrm{M}^{+}\right], 262$ (100), 233 (3), 204 (4). HRMS: $m / z$ calcd for $\mathrm{C}_{23} \mathrm{H}_{23} \mathrm{NO}_{2}\left[\mathrm{M}^{+}\right]$: 345.1729; found: 345.1746 .

Murrayamine G (8). A solution of ( \pm )-mahanimbicine $[( \pm)-4]$ $(63.8 \mathrm{mg}, 193 \mu \mathrm{mol})$ and CSA $(22.8 \mathrm{mg}, 98.2 \mu \mathrm{mol})$ in toluene $(9 \mathrm{~mL})$ was stirred at room temperature for $6 \mathrm{~d}$. An additional portion of CSA $(22.4 \mathrm{mg}, 96.4 \mu \mathrm{mol})$ was added and the mixture was stirred at room temperature for $7 \mathrm{~d}$ and at $70{ }^{\circ} \mathrm{C}$ for $3 \mathrm{~d}$ (total reaction time: $16 \mathrm{~d}$ ). The mixture was diluted with diethyl ether and washed with an aqueous solution of sodium hydrogencarbonate and brine. The aqueous layers were extracted with diethyl ether. Subsequently, the combined organic layers were dried over sodium sulfate and the solvent was evaporated. Purification of the residue by column chromatography on silica gel (pentane-dichloromethane-ethyl acetate, gradient elution, $60: 1: 0.2$ to $17: 1: 0.2)$ provided murrayamine-G (8) as colourless crystals, yield: $41.4 \mathrm{mg}(65 \%)$, m.p. $166-169{ }^{\circ} \mathrm{C}$ (ref. 9: 173-176 ${ }^{\circ} \mathrm{C}$ ). $(\mathrm{MeOH}): \lambda=219$ (sh), 241, 257 (sh), 262, 307, 323 (sh) nm. Fluorescence $(\mathrm{MeOH}): \lambda_{\text {ex }}$ $=307 \mathrm{~nm}, \lambda_{\mathrm{em}}=358 \mathrm{~nm}$. IR (ATR): $\nu=3463$, 3074, 2963, 2920, 2862, 1613, 1519, 1481, 1445, 1420, 1378, 1312, 1297, 1217, 1172, 1159, 1100, 1027, 989, 963, 915, 888, 816, 798, 744, 658, 621, 584, $559 \mathrm{~cm}^{-1} .{ }^{1} \mathrm{H}$ NMR (500 MHz, $\left.\mathrm{CDCl}_{3}\right): \delta=1.43(\mathrm{~s}$, $3 \mathrm{H}), 1.48-1.50$ (m, $1 \mathrm{H}), 1.50$ (s, $3 \mathrm{H}), 1.61-1.67$ (m, $2 \mathrm{H}), 1.94$ (dt, $J=12.9,3.1 \mathrm{~Hz}, 1 \mathrm{H}), 2.03(\mathrm{dd}, J=12.9,2.7 \mathrm{~Hz}, 1 \mathrm{H}), 2.11$ $(\mathrm{dt}, J=9.3,2.3 \mathrm{~Hz}, 1 \mathrm{H}), 2.49(\mathrm{~s}, 3 \mathrm{H}), 2.58(\mathrm{~m}, 1 \mathrm{H}), 3.41(\mathrm{~m}$, $1 \mathrm{H}), 4.73(\mathrm{~s}, 1 \mathrm{H}), 4.81(\mathrm{t}, J=1.5 \mathrm{~Hz}, 1 \mathrm{H}), 6.72(\mathrm{~d}, J=8.5 \mathrm{~Hz}$, $1 \mathrm{H}), 7.11(\mathrm{dd}, J=8.1,1.2 \mathrm{~Hz}, 1 \mathrm{H}), 7.22(\mathrm{~d}, J=8.1 \mathrm{~Hz}, 1 \mathrm{H})$, 7.70 (br s, $1 \mathrm{H}), 7.71$ (s, $1 \mathrm{H}), 7.73$ (d, $J=8.5 \mathrm{~Hz}, 1 \mathrm{H}) .{ }^{13} \mathrm{C} \mathrm{NMR}$ and DEPT (125 MHz, $\left.\mathrm{CDCl}_{3}\right): \delta=21.41\left(\mathrm{CH}_{3}\right), 21.57\left(\mathrm{CH}_{3}\right)$, $23.00\left(\mathrm{CH}_{2}\right), 28.89\left(\mathrm{CH}_{3}\right), 35.95(\mathrm{CH}), 37.40\left(\mathrm{CH}_{2}\right), 39.67\left(\mathrm{CH}_{2}\right)$, $48.54(\mathrm{CH}), 74.08$ (C), 105.67 (C), $108.70(\mathrm{CH}), 109.89(\mathrm{CH})$, $112.09\left(\mathrm{CH}_{2}\right), 115.36(\mathrm{C}), 119.08(\mathrm{CH}), 119.22(\mathrm{CH}), 124.40(\mathrm{C})$, 125.05 (CH), 128.65 (C), 137.60 (C), 140.08 (C), 149.89 (C), 155.17 (C). EI-MS (70 eV): $m / z(\%)=331(80)\left[\mathrm{M}^{+}\right], 316$ (11), 248 (100), 210 (9). HRMS: $m / z$ calcd for $\mathrm{C}_{23} \mathrm{H}_{25} \mathrm{NO}\left[\mathrm{M}^{+}\right]$: 331.1936; found: 331.1936. Elemental analysis calcd for $\mathrm{C}_{23} \mathrm{H}_{25} \mathrm{NO}$ : C 83.34, H 7.60, N 4.23; found: C 83.03, H 7.79, N 4.18\%. 
Isomurrayazoline (9). A solution of ( \pm )-mahanimbicine $[( \pm)-4](40.5 \mathrm{mg}, 122 \mu \mathrm{mol})$ and CSA $(2.3 \mathrm{mg}, 10 \mu \mathrm{mol})$ in hexane $(12 \mathrm{~mL})$ was stirred at room temperature for $11.5 \mathrm{~d}$. The mixture was diluted with diethyl ether and washed with an aqueous solution of sodium hydrogencarbonate and brine. The aqueous layers were extracted with diethyl ether, the combined organic layers were dried over sodium sulfate and the solvent was evaporated. Purification of the residue by column chromatography on silica gel (pentane-dichloromethane-ethyl acetate, gradient elution, 59:1:0.2 to $16: 1: 0.2)$ provided a mixture of murrayamine $\mathrm{G}(8)$ and isomurrayazoline (9) as colourless solid, yield: $28.4 \mathrm{mg}(70 \%)$, ratio of $8: 9=1: 1$ (determined by the ${ }^{1} \mathrm{H}$ NMR spectrum). The two isomers were separated by preparative HPLC on a Grace Vydac C8 column $(208 \mathrm{TP} 1050,50 \times 250 \mathrm{~mm})$, gradient elution with $55 \mathrm{~mL} \mathrm{~min}{ }^{-1}$ (THF- $\mathrm{H}_{2} \mathrm{O}, 20-70 \%$ THF in $25 \mathrm{~min}$ ) to afford murrayamine-G (8) as colourless crystals (spectroscopic data: see above) and isomurrayazoline (9) as colourless solid, m.p. $224-227{ }^{\circ} \mathrm{C}$ (ref. 10: $269-270{ }^{\circ} \mathrm{C}$ ). UV (MeOH): $\lambda=239$ (sh), 246, 262 (sh), 307, 327 (sh), 340 (sh) nm. Fluorescence $(\mathrm{MeOH}): \lambda_{\mathrm{ex}}=246 \mathrm{~nm}, \lambda_{\mathrm{em}}=366 \mathrm{~nm}$. IR (ATR): $\nu=3047,2965$, 2922, 2900, 1631, 1592, 1446, 1416, 1347, 1307, 1288, 1221, 1204, 1180, 1152, 1134, 1087, 1068, 1037, 985, 941, 914, 882, $855,801,787,771,744,680,625,602,593,556 \mathrm{~cm}^{-1} .{ }^{1} \mathrm{H}$ NMR $\left(500 \mathrm{MHz}, \mathrm{CDCl}_{3}\right): \delta=0.13-0.21(\mathrm{~m}, 1 \mathrm{H}), 1.26-1.33(\mathrm{~m}, 1 \mathrm{H})$, $1.28(\mathrm{~s}, 3 \mathrm{H}), 1.44(\mathrm{~s}, 3 \mathrm{H}), 1.47-1.54(\mathrm{~m}, 1 \mathrm{H}), 1.68(\mathrm{ddd}, J=$ 15.3, 6.8, 3.2 Hz, $1 \mathrm{H}), 1.90$ (s, $3 \mathrm{H}), 1.90-1.93$ (m, $1 \mathrm{H}), 1.98$ (ddd, $J=11.1,5.5,2.4 \mathrm{~Hz}, 1 \mathrm{H}$ ), 2.41 (ddd, $J=13.3,5.3,3.4 \mathrm{~Hz}$, $1 \mathrm{H}), 2.49(\mathrm{~s}, 3 \mathrm{H}), 3.29$ (d, $J=4.6 \mathrm{~Hz}, 1 \mathrm{H}), 6.64(\mathrm{~d}, J=8.2 \mathrm{~Hz}$, $1 \mathrm{H}), 7.09$ (dd, $J=8.3,1.3 \mathrm{~Hz}, 1 \mathrm{H}), 7.38(\mathrm{~d}, J=8.3 \mathrm{~Hz}, 1 \mathrm{H})$, $7.58(\mathrm{~d}, J=8.2 \mathrm{~Hz}, 1 \mathrm{H}), 7.72(\mathrm{~m}, 1 \mathrm{H}) .{ }^{13} \mathrm{C}-\mathrm{NMR}$ and DEPT (125 MHz, $\left.\mathrm{CDCl}_{3}\right): \delta=21.31\left(\mathrm{CH}_{3}\right), 21.70\left(\mathrm{CH}_{2}\right), 22.91\left(\mathrm{CH}_{3}\right)$, $28.12(\mathrm{CH}), 29.15\left(\mathrm{CH}_{3}\right), 30.14\left(\mathrm{CH}_{3}\right), 36.06\left(\mathrm{CH}_{2}\right), 36.56\left(\mathrm{CH}_{2}\right)$, 48.35 (CH), 60.47 (C), 76.26 (C), 107.73 (C), 108.87 (CH), 113.16 $(\mathrm{CH}), 114.51(\mathrm{C}), 119.00(\mathrm{CH}), 120.16(\mathrm{CH}), 124.10(\mathrm{CH}), 127.50$ (C), 128.82 (C), 138.92 (C), 144.32 (C), 156.37 (C). EI-MS (70 $\mathrm{eV}): m / z(\%)=331(99)\left[\mathrm{M}^{+}\right], 316(100), 288$ (15), 248 (63). Elemental analysis calcd for $\mathrm{C}_{23} \mathrm{H}_{25} \mathrm{NO}$ : $\mathrm{C}$ 83.34, $\mathrm{H} 7.60, \mathrm{~N}$ 4.23; found: C 83.00, H 7.89, N 4.26\%.

\section{Acknowledgements}

We thank Micha P. Krahl for experimental support.

\section{Notes and references}

1 (a) D. P. Chakraborty and S. Roy, in Progress in the Chemistry of Organic Natural Products, ed. W. Herz, H. Grisebach, G. W. Kirby, W. Steglich and C. Tamm, Springer-Verlag, Wien, 1991, vol. 57, p. 71; (b) H.-J. Knölker and K. R. Reddy, Chem. Rev., 2002, 102, 4303.

2 (a) H.-J. Knölker and K. R. Reddy, in The Alkaloids, ed. G. A. Cordell, Academic Press, Amsterdam, 2008, vol. 65, p. 1; (b) A. W. Schmidt, K. R. Reddy and H.-J. Knölker, Chem. Rev., 2012, 112, 3193.

3 (a) D. P. Chakraborty, B. K. Barman and P. K. Bose, Sci. Cult., 1964, 30, 445; (b) N. L. Dutta and C. Quasim, Indian J. Chem., 1969, 7, 307; (c) S. P. Kureel, R. S. Kapil and S. P. Popli, Chem. Ind., 1970, 1262; (d) B. S. Joshi, V. N. Kamat, D. H. Gawad and T. R. Govindachari, Phytochemistry, 1972, 11, 2065.

4 (a) D. P. Chakraborty, K. C. Das and P. K. Bose, Sci. Cult., 1966, 32, 83; (b) N. S. Narasimhan, M. V. Paradkar and V. P. Chitguppi, Tetrahedron Lett., 1968, 9, 5501; (c) N. S. Narasimhan, M. V. Paradkar, V. P. Chitguppi and S. L. Kelkar, Indian J. Chem., 1975, 13, 993; (d) N. S. Narasimhan, M. V. Paradkar and A. M. Gokhale, Indian J. Chem., 1976, 14B, 329; (e) H. Furukawa, T. S. Wu, T. Ohta and C.-S. Kuoh, Chem. Pharm. Bull., 1985, 33, 4132; $(f)$ K. M. Meragelman, T. C. McKee and M. R. Boyd, J. Nat. Prod., 2000, 63, 427.

5 S. P. Kureel, R. S. Kapil and S. P. Popli, Chem. Ind., 1970, 958. 6 W. M. Bandaranayake, M. J. Begley, B. O. Brown, D. G. Clarke, L. Crombie and D. A. Whiting, J. Chem. Soc., Perkin Trans. 1, 1974, 998.

7 B. S. Joshi, V. N. Kamat and D. H. Gawad, Tetrahedron, 1970, 26, 1475.

8 T.-S. Wu, M.-L. Wang, P.-L. Wu, C. Ito and H. Furukawa, Phytochemistry, 1996, 41, 1433.

9 T.-S. Wu, M.-L. Wang and P.-L. Wu, Phytochemistry, 1996, 43, 785.

10 L. Bhattacharya, S. K. Roy and D. P. Chakraborty, Phytochemistry, 1982, 21, 2432.

11 (a) H.-J. Knölker and C. Hofmann, Tetrahedron Lett., 1996, 37, 7947; (b) K. K. Gruner and H.-J. Knölker, Org. Biomol. Chem., 2008, 6, 3902; (c) K. K. Gruner, T. Hopfmann, K. Matsumoto, A. Jäger, T. Katsuki and H.-J. Knölker, Org. Biomol. Chem., 2011, 9, 2057.

12 R. Hesse, K. K. Gruner, O. Kataeva, A. W. Schmidt and H.-J. Knölker, Chem. - Eur. J., 2013, 19, 14098.

13 V. P. Kumar, K. K. Gruner, O. Kataeva and H.-J. Knölker, Angew. Chem., Int. Ed., 2013, 52, 11073.

14 (a) R. Hesse, A. Jäger, A. W. Schmidt and H.-J. Knölker, Org. Biomol. Chem., 2014, 12, 3866; (b) K. K. Gruner, O. Kataeva, A. W. Schmidt and H.-J. Knölker, Chem. - Eur. J., 2014, 20, 8536; (c) R. Hesse, O. Kataeva, A. W. Schmidt and H.-J. Knölker, Chem. - Eur. J., 2014, 20, DOI: 10.1002/ chem.201403645.

15 (a) H.-J. Knölker and N. O'Sullivan, Tetrahedron, 1994, 50, 10893; (b) H.-J. Knölker, Curr. Org. Synth., 2004, 1, 309; (c) R. Forke, A. Jäger and H.-J. Knölker, Org. Biomol. Chem., 2008, 6, 2481; (d) R. Forke, M. P. Krahl, F. Däbritz, A. Jäger and H.-J. Knölker, Synlett, 2008, 1870; (e) H.-J. Knölker, Chem. Lett., 2009, 38, 8; (f) I. Bauer and H.-J. Knölker, Top. Curr. Chem., 2012, 309, 203; $(g)$ T. Gensch, M. Rönnefahrt, R. Czerwonka, A. Jäger, O. Kataeva, I. Bauer and H.-J. Knölker, Chem. Eur. J., 2012, 18, 770; (h) L. Huet, R. Forke, A. Jäger and H.-J. Knölker, Synlett, 2012, 1230. 
16 M. P. Krahl, A. Jäger, T. Krause and H.-J. Knölker, Org. Biomol. Chem., 2006, 4, 3215.

17 (a) J. F. Hartwig, Angew. Chem., Int. Ed., 1998, 37, 2046; (b) M. D. Charles, P. Schultz and S. L. Buchwald, Org. Lett., 2005, 7, 3965; (c) D. S. Surry and S. L. Buchwald, Angew. Chem., Int. Ed., 2008, 47, 6338.

18 (a) A. K. Chakravarty, T. Sarkar, K. Masuda, T. Takey, H. Doi, E. Kotani and K. Shiojima, Indian J. Chem., 2001, 40B, 484; (b) S. Cheenpracha and S. Laphookhieo, Phytochem. Lett., 2011, 4, 187; (c) J. T. Kuethe and K. G. Childers, Adv. Synth. Catal., 2008, 350, 1577.

19 J. D. Godfrey Jr., R. H. Mueller, T. C. Sedergran, N. Soundararajan and V. J. Colandrea, Tetrahedron Lett., 1994, 35, 6405.

20 (a) I. Iwai and J. Ide, Chem. Pharm. Bull., 1962, 10, 926; (b) I. Iwai and J. Ide, Chem. Pharm. Bull., 1963, 11, 1042; (c) J. Zsindely and H. Schmid, Helv. Chim. Acta, 1968, 51, 1510; (d) J. Hlubucek, E. Ritchie and W. C. Taylor, Tetra- hedron Lett., 1969, 10, 1369; (e) P. E. Brown and R. A. Lewis, J. Chem. Soc., Perkin Trans. 1, 1992, 573.

21 (a) J. Tsuji, T. Sugiura and I. Minami, Synthesis, 1987, 603; (b) S. Yamaguchi, M. Maekawa, Y. Murayama, M. Miyazawa and Y. Hirai, Tetrahedron Lett., 2004, 45, 6971; (c) S. Yamaguchi, M. Nedachi, M. Maekawa, Y. Murayama, M. Miyazawa and Y. Hirai, J. Heterocycl. Chem., 2006, 43, 29.

22 A. Yasuhara and T. Sakamoto, Tetrahedron Lett., 1998, 39, 595.

23 (a) T. A. Choi, R. Czerwonka, W. Fröhner, M. P. Krahl, K. R. Reddy, S. G. Franzblau and H.-J. Knölker, ChemMedChem, 2006, 1, 812; (b) T. A. Choi, R. Czerwonka, R. Forke, A. Jäger, J. Knöll, M. P. Krahl, T. Krause, K. R. Reddy, S. G. Franzblau and H.-J. Knölker, Med. Chem. Res., 2008, 17,374 .

24 H. E. Gottlieb, V. Kotlyar and A. Nudelman, J. Org. Chem., 1997, 62, 7512. 